\title{
Magnetic Catalysis of Chiral Symmetry Breaking: A Holographic Prospective
}

\author{
Veselin Filev ${ }^{1}$ and Radoslav Rashkov ${ }^{2,3}$ \\ ${ }^{1}$ School of Theoretical Physics, Dublin Institute for Advanced Studies, 10 Burlington Road, \\ Dublin 4, Ireland \\ ${ }^{2}$ Institute for Theoretical Physics, Vienna University of Technology, Wiedner Hauptstr. 8-10, \\ 1040 Vienna, Austria \\ ${ }^{3}$ Department of Physics, Sofia University, Bulgaria \\ Correspondence should be addressed to Radoslav Rashkov, rash@phys.uni-sofia.bg
}

Received 29 April 2010; Accepted 16 June 2010

Academic Editor: Carlos Nunez

Copyright (c) 2010 V. Filev and R. Rashkov. This is an open access article distributed under the Creative Commons Attribution License, which permits unrestricted use, distribution, and reproduction in any medium, provided the original work is properly cited.

We review a recent investigation of the effect of magnetic catalysis of mass generation in holographic Yang-Mills theories. We aim at a self-contained and pedagogical form of the review. We provide a brief field theory background and review the basics of holographic flavordynamics. The main part of the paper investigates the influence of external magnetic field to holographic gauge theories dual to the D3/D5- and D3/D7-brane intersections. Among the observed phenomena are the spontaneous breaking of a global internal symmetry, Zeeman splitting of the energy levels, and the existence of pseudo, Goldstone modes. An analytic derivation of the GellMann-Oaks-Renner relation for the D3/D7 set up is reviewed. In the D3/D5 case, the pseudoGoldstone modes satisfy nonrelativistic dispersion relation. The studies reviewed confirm the universal nature of the magnetic catalysis of mass generation.

\section{Introduction}

An important concept in our attempt to describe the structure of our physical reality dating back to Democritus is the atomic principle, namely, the idea that macroscopic bodies are build out of fundamental particles. In modern perspective, we are interested in studying the basic interactions between the building blocks of matter. It is experimentally well established that there are four fundamental interactions: electromagnetic, strong and weak interactions as well as gravity. Despite the remarkable success of the Standard Model of particle physics unifying the first four interactions, it still remains a challenge to come up with a consistent quantum theory of gravity. At present, one of the most promising directions towards a unified theory of the fundamental interactions lies in the framework of string theory. 
Historically, string theory emerged as an attempt to describe the strong interactions by what was called dual resonance models. However, shortly after its discovery, Quantum Chromodynamics (QCD), which is a SU(3) Yang-Mills gauge theory, superseded it. The matter degrees of QCD, consist of quarks which are in the fundamental representation of the gauge group, while the interaction between the fundamental fields is being mediated by the gluons which are the gauge fields of the theory thus transforming in the adjoint representation of $S U(3)$.

A remarkable property of QCD is the fact that it is asymptotically free, meaning that at large energy scales, or equivalently at short distances, it has a vanishing coupling constant. This makes QCD perturbatively accessible at ultraviolet. However, the low energy regime of the theory is quite different. At low energy, QCD is strongly coupled, the interaction force between the quarks grows immensely and they are bound together; they form hadrons. This phenomenon is called confinement. Additional property of the low energy dynamics of QCD is the formation of a quark condensate which mixes the left and right degrees of the fundamental matter and leads to a breaking of their chiral symmetry. It is extremely hard to examine the properties of the strongly coupled low energy regime of QCD, since the usual perturbative techniques are not applicable.

The AdS/CFT correspondence [1, 2], as we will describe in detail in Section 3.1 of this review, is a powerful analytic tool providing a nonperturbative dual description of non-abelian gauge theories, in terms of string theory defined on a proper gravitational background. An important extension of the correspondence making it relevant to the description of flavored Yang-Mills theories was the introduction of fundamental matter via the introduction of probe flavor branes [3]. The most understood case is in the limit when the number of different flavors is much less than the number of colors. This corresponds to the quenched approximation on gauge theory side and the probe approximation on supergravity side of the correspondence. We will review more details about the way the AdS/CFT dictionary works in Section 3.2.

Despite its great potential direct application of the AdS/CFT, correspondence to realistic non-abelian gauge theories such as QCD remains a challenge. A major limitation is that realistic field theories do not seem to have simple holographic backgrounds. Furthermore, there are indications that most realistic gauge theories do not even pose exact holographically dual geometries. Nevertheless, applications of the AdS/CFT correspondence are still possible. One plausible direction is the investigation of non-abelian gauge theories exhibiting universal behaviour. Particularly interesting is to analyze the phase structure of strongly coupled Yang-Mills theories. An example of such application of the holographic approach is the study of properties of strongly coupled quark-gluon plasmas [4].

Another possible direction is the study of phenomena known to have a universal nature. An important example is the phenomenon of mass generation in an external magnetic field. This phenomenon has been extensively studied in the conventional field theory literature [5-8]. The effect was shown to be model independent and therefore insensitive to the microscopic physics underlying the low-energy effective theory. The essence of this effect is the dimensional reduction $\mathrm{D} \rightarrow \mathrm{D}-2(3+1 \rightarrow 1+1)$ in the dynamics of fermion pairing in a magnetic field. Magnetic catalysis of mass generation has been demonstrated in various $1+2$ and $1+3$ dimensional field theories. Given the universal nature of this effect, it is natural to explore this phenomenon in the context of holographic gauge theories. In this review, we focus on such studies for holographic gauge theories dual to the Dp/Dq-brane intersection. 
The structure of the review is as follows.

In Section 2, we provide a short field theory background. In the first subsection, we describe the properties of flavored $S U\left(N_{c}\right)$ Yang-Mills theory focusing on the global symmetries of the Lagrangian. We remind the reader about some basics of the phenomenon of Chiral Symmetry breaking. We describe the effective field theory approach to that of Chiral Symmetry breaking and provide a brief derivation of the famous Gell-Mann-Oaks-Renner relation [9]. In the second subsection, we review the mechanism of the chiral symmetry breaking due to the presence of an external magnetic field [5].

Section 3 of this review is dedicated to the AdS/CFT correspondence and its extension to include matter in the fundamental representation of the gauge group. In the first subsection we outline the main ideas that lead to the formulation of the Maldacena's conjecture [1,2]. We discuss some qualitative and quantitative aspects of the correspondence and provide a brief description about the way the AdS/CFT dictionary operates. The second subsection focuses on the addition of flavor degrees of freedom to the correspondence. We review the approach of [3] and provide some basic extracts from the AdS/CFT dictionary, which will be important for the studies presented in Section 4.

Section 4 is the main part of the review. We present the studies of the influence of external magnetic field on holographic gauge theories dual to the D3/D5- and the D3/D7branes intersection performed in [10-13]. In the case of the D3/D7 system, we review the general properties of the holographic set up and the way chiral symmetry breaking is realized as a separation of the color and flavor branes in the infrared. We review the properties of the light meson spectrum of the theory and uncover Zeeman splitting of the energy levels as well as the existence of Goldstone modes corresponding to the spontaneously broken Chiral Symmetry. In the limit of small bare masses review, the analytic derivation of the Gell-Mann-Oaks-Renner relation obtained in [12] from dimensional reduction of the eightdimensional effective action of the probe D7-brane. We also review the analogous studies of the D3/D5 system. Again there are mass generation, Zeeman splitting, and Goldstone modes. Interestingly, the broken Lorentz invariance in this case leads to nonrelativistic dispersion relations.

We end with a brief summary of the presented material and a short discussion in the conclusion section of the review.

\section{Field Theory Preliminaries}

In this section, we provide a basic field theory background. Our goal is to remind the reader about some of the properties of strongly coupled flavored Yang-Mills theories, in particular, their global symmetries and the corresponding spontaneous symmetry breaking. We outline the effective field theory description of Chiral Symmetry breaking and provide a brief derivation of the Gell-Mann-Oaks-Renner relation [9]. We also provide a short review of the effect of magnetic catalysis of mass generation.

\subsection{Flavored Yang-Mills Theory and Chiral Symmetry Breaking}

Flavored Yang-Mills Theory

The lagrangian of four-dimensional pure $S U\left(N_{c}\right)$ Yang-Mills theory coupled to $N_{f}$ flavors of fermionic fields is given by 


$$
\begin{gathered}
\mathcal{L}=-\frac{1}{4 g_{Y M}^{2}} \operatorname{Tr}\left[F_{\mu \nu} F^{\mu \nu}\right]+\frac{\theta}{32 \pi^{2}} \operatorname{Tr}\left[F_{\mu \nu} \tilde{F}^{\mu \nu}\right]+\sum_{i=1}^{N_{f}} \bar{\psi}^{i}\left(i \gamma^{\mu} D_{\mu}-m_{i}\right) \psi^{i}, \\
F_{\mu \nu}=\partial_{\mu} A_{\nu}-\partial_{\nu} A_{\mu}-i\left[A_{\mu}, A_{\nu}\right], \quad D_{\mu}=\partial_{\mu}-i A_{\mu}, \quad \widetilde{F}^{\mu \nu}=\frac{1}{2} \varepsilon^{\mu \nu \rho \sigma} F_{\rho \sigma} .
\end{gathered}
$$

The first term in (2.1) is a dynamical term for the gauge field $A_{\mu}$. The second term (the socalled $\theta$-term) is topological and is related to the Pontryagin index of $A_{\mu}$. The parameter $\theta$ ranges from 0 to $2 \pi$ and parametrizes topologically distinct sectors of the theory. The last term in (2.1) describes the matter (fundamental) fields of the theory. Let us look closely at the last term. If one defines the left and right fermionic fields $\psi_{L, R}=1 / 2\left(1 \pm \gamma^{5}\right) \psi$, it can be written as

$$
\mathcal{L}_{f}=\sum_{i=1}^{N_{f}}\left(\bar{\psi}_{L}^{i} i \gamma^{\mu} D_{\mu} \psi_{L}^{i}+\bar{\psi}_{R}^{i} i \gamma^{\mu} D_{\mu} \psi_{R}^{i}-m_{i}\left(\bar{\psi}_{L}^{i} \psi_{R}^{i}+\bar{\psi}_{R}^{i} \psi_{L}^{i}\right)\right)
$$

It is clear that the mass of the matter fields $m_{i}$ can be interpreted as a coupling between the left and right fields $\psi_{L / R}$. Therefore, at vanishing $m_{i}$ we have two distinct sets of $N_{f}$ fermionic fields and at classical level the theory has a global $U\left(N_{f}\right)_{L} \times U\left(N_{f}\right)_{R}$ symmetry. It is instructive to split the global symmetry to

$$
U\left(N_{f}\right)_{L} \times U\left(N_{f}\right)_{R}=S U\left(N_{f}\right)_{V} \times S U\left(N_{f}\right)_{A} \times U(1)_{V} \times U(1)_{A}
$$

Let us focus first on the abelian symmetry. In infinitesimal form we have the transformations:

$$
\begin{array}{lll}
\delta \psi_{L}=-i \alpha \psi_{L}, & \delta \psi_{R}=-i \alpha \psi_{R}, & \text { for } U(1)_{V} \\
\delta \psi_{L}=-i \alpha \psi_{L}, & \delta \psi_{R}=+i \alpha \psi_{R}, & \text { for } U(1)_{A} .
\end{array}
$$

Transformation (2.5) is just a rigid $U(1)$-gauge transformation and correspond to some quantum number. We will not be interested in breaking gauge symmetries in these notes; this is why we focus on the transformation (2.5).

\section{Anomalous Chiral Symmetry}

In terms of the fields $\psi, \bar{\psi}$, transformation (2.5) can be written as

$$
\delta \psi=-i \alpha \gamma^{5} \psi, \quad \delta \bar{\psi}=-i \alpha \bar{\psi} \gamma^{5}
$$

The corresponding Noether current is given by

$$
j^{\mu 5}=\bar{\psi} \gamma^{\mu} \gamma^{5} \psi,
$$

and is conserved upon applying the equations of motion. Clearly a nonzero fermionic condensate $\langle\bar{\psi} \psi\rangle$ would break the Chiral transformation (2.6). Naively one would expect 
the existence of a corresponding Goldstone boson. This is the famous $\eta^{\prime}$-meson in QCD. However, it turns out that the measure of the path integral has a non-zero Jacobian under the transformation (2.6), and the axial current defined in (2.7) is anomalous. In fact, one can show that the anomaly is given by (look at [14, pages 185-192] for a brief derivation)

$$
\partial_{\mu} j^{\mu 5}=-\frac{1}{16 \pi^{2}} \operatorname{Tr}\left[F_{\mu \nu} \widetilde{F}^{\mu \nu}\right]
$$

and the chiral transformation can be absorbed into a redefinition of the theta angle of the theory $\theta \rightarrow \theta-2 \alpha$. This suggests that the mass of the $\eta^{\prime}$-meson can be related to the topological susceptibility of the theory $\chi_{\mathrm{YM}}=\partial^{2} \varepsilon_{\mathrm{vac}} /\left.\partial^{2} \theta\right|_{N_{f}=0}$. For a canonically normalized $\eta^{\prime}$-field, one can obtain the following Witten-Veneziano formula $[15,16]$ :

$$
m_{\eta^{\prime}}^{2}=\frac{4 N_{f}}{f_{\eta^{\prime}}^{2}} X_{\mathrm{YM}} \propto \frac{N_{f}}{N_{c}},
$$

where we have used the $N_{c}$ dependence of $f_{\eta^{\prime}}$ for large $N_{c}, f_{\eta^{\prime}} \propto \sqrt{N_{c}}$ [17].

The fact that $m_{\eta^{\prime}}^{2} \propto N_{f} / N_{c}$ has an important consequences for the large $N_{c}$ limit of the theory. It suggests that if the number of flavors is $N_{f} \ll N_{c}$ (the so-called quenched approximation), the mass of the $\eta^{\prime}$-meson is effectively zero and the anomalous $U(1)_{A}$ axial symmetry is restored. This result is essential for the holographic studies that we will review in Section 4. In fact, the holographic supersymmetric gauge theory that we consider has an anomalous $U(1) R$-symmetry, which mimics the anomalous $U(1)_{A}$ symmetry (2.6). It is the spontaneous breaking of this symmetry under external magnetic field that has been explored in the holographic set up presented in Section 4.1.

\section{Nonsinglet Chiral Symmetry}

Let us now focus on the non-abelian part of the global symmetry (2.3). We will be interested in the dynamical breaking of this symmetry by a nonvanishing fundamental condensate $\langle\bar{\psi} \psi\rangle$. Clearly only the axial $S U\left(N_{f}\right)_{A}$ is broken by the fundamental condensate. Goldstone theorem suggests the existence of $N_{f}^{2}-1$ goldstone fields. In $N_{f}=3$ Quantum Chromodynamics, these are the $\pi_{ \pm}, \pi_{0}, K_{ \pm}, K_{0}, \bar{K}_{0}$, and $\eta$ mesons.

An important extension of this discussion is the case when the mass of the fundamental fields is not vanishing but is still a small parameter with respect to some relevant energy scale. In this case, the Chiral Symmetry is an approximate symmetry of the theory and the corresponding goldstone particles (mesons) acquire small masses. At leading order, there is an important relation between the mass of the mesons, the bare mass of the fundamental fermions, and the fundamental condensate, namely, the Gell-Mann-Oaks-Renner relation [9]. Since we will be interested in verifying this relation via holographic techniques in Section 4, let us provide a brief derivation using an effective field theory description. 


\section{Effective Chiral Lagrangian and the Gell-Mann-Oaks-Renner Relation}

In what follows we will treat the fundamental condensate of the theory as an order parameter of dynamically broken Chiral Symmetry along the lines of [14]. Let us define a condensate matrix as

$$
\Sigma_{0}^{i j}=\left\langle\bar{\psi}_{L}^{i} \psi_{R}^{j}\right\rangle_{0}
$$

Nonbreaking of the vector symmetry implies that the matrix order parameter $\Sigma^{i j}$ can be brought into the following form:

$$
\Sigma_{0}^{i j}=\frac{1}{2} \delta^{i j} \Sigma_{0}
$$

by the group transformation (2.3). Here $\Sigma_{0}$ is in general a complex scalar. Now fluctuations of the order parameter (2.11) will be described by a unitary matrix $U(x) \in S U\left(N_{f}\right)_{A}$ :

$$
\Sigma(x)^{i j}=\frac{1}{2} \Sigma_{0} U(x)^{i j}
$$

as well as a fluctuations of the phase of $\Sigma_{0}$ parametrized by elements of $U(1)_{A}$. Let us focus on the non-abelian case first. It is convenient to express $U(x)$ as an exponential

$$
U(x)=\exp \left\{\frac{2 i \pi^{a}(x) t^{a}}{f_{\pi}^{2}}\right\}
$$

where $\pi^{a}$ are the physical meson fields, $f_{\pi}$ is a constant of dimension of mass (the pion's decay constant in Quantum Chromodynamics), and $t^{a}$ are the generators of $S U\left(N_{f}\right)_{A}$. To fix the exact form of the effective lagrangian, note that there is a unique invariant structure involving two derivatives:

$$
\mathcal{L}_{\mathrm{eff}}^{(\mathrm{kin})}=\frac{f_{\pi}^{2}}{4} \operatorname{Tr}\left[\partial_{\mu} U \partial^{\mu} U^{\dagger}\right]
$$

To leading order in $\pi^{a}$, we obtain

$$
\mathcal{L}_{\mathrm{eff}}^{(2, \text { kin })}=\frac{1}{2} \partial_{\mu} \pi^{a} \partial^{\mu} \pi^{a}+\cdots
$$

and hence we have a canonically normalized bosonic field. In order to fix the mass term of our pseudo-Goldstone fields, let us note that the mass term in (2.2) can be traded for

$$
\mathcal{L}_{\text {eff }}^{m}=-\operatorname{Re}[\operatorname{Tr}\{\mathcal{M} \Sigma(x)\}],
$$


where we have defined the mass matrix $\mathcal{M}=\left\|m_{i} \delta^{i j}\right\|$. Now using (2.12) and (2.13) one arrives at

$$
\mathcal{L}_{\mathrm{eff}}^{m}=\text { const }+\frac{\operatorname{Re}\left[\Sigma_{0}\right]}{f_{\pi}^{2}}\left(\sum_{i=1}^{N_{f}} m_{i}\right) \pi^{a} \pi^{a}+O\left(\pi^{3}\right) .
$$

Therefore, to quadratic order our effective action is given by

$$
\mathcal{L}_{\mathrm{eff}}^{(2)}=\frac{1}{2} \partial_{\mu} \pi^{a} \partial^{\mu} \pi^{a}+\frac{\operatorname{Re}\left[\Sigma_{0}\right]}{f_{\pi}^{2}}\left(\sum_{i=1}^{N_{f}} m_{i}\right) \pi^{a} \pi^{a} .
$$

Equation (2.18) implies the following expression for the mass of the meson fields $\pi^{a}$ :

$$
M_{\pi}^{2}=-\frac{2 \operatorname{Re}\left[\Sigma_{0}\right]}{f_{\pi}^{2}}\left(\sum_{i=1}^{N_{f}} m_{i}\right)=-\frac{2\langle\bar{\psi} \psi\rangle}{f_{\pi}^{2}} m,
$$

where in the last equality we have used is $\langle\bar{\psi} \psi\rangle=N_{f} \operatorname{Re}\left[\Sigma_{0}\right]$ and we defined

$$
m=\frac{1}{N_{f}} \sum_{i=1}^{N_{f}} m_{i}
$$

Equation (2.19) is the famous Gell-Mann-Oaks-Renner relation [9]. Using similar arguments, one can obtain similar expression for the mass of the $\eta^{\prime}$-meson corresponding to the spontaneous breaking of the $U(1)_{A}$ Chiral Symmetry (which is nonanomalous in the quenched approximation $N_{f} \ll N_{c}$ ).

\subsection{Magnetic Catalysis of Mass Generation in Field Theory}

In this subsection, we will review the mechanism of the chiral symmetry breaking due to the presence of an external magnetic field. We will follow closely the outline provided in $[18,19]$.

To start with, let us make a few comments on the general properties of the chiral fermions in a constant magnetic field turned on in $x^{3}$ direction of the $4 \mathrm{~d}$ spacetime. The lagrangian of a relativistic fermion is of the standard form

$$
\mathcal{L}=\frac{1}{2}\left[\bar{\Psi},\left(i \gamma^{\mu} D_{\mu}-m\right) \Psi\right]
$$

where the covariant derivative is given by

$$
D_{\mu}=\partial_{\mu}-i e A_{\mu}^{e x t}, \quad A_{\mu}^{e x t}=-B x_{2} \delta_{\mu, 1} .
$$


One of the most important characteristic of the system is its spectrum, or the so-called Landau levels, which can be easily obtained from the above lagrangian

$$
E_{n}\left(k_{3}\right)= \pm \sqrt{m^{2}+2|e B| n+k_{3}^{2}}, \quad n=0,1,2, \ldots
$$

First of all, one can immediately see the degeneracy of the Landau levels. The energy is parametrized by a continuous parameter $k_{3}$, the momentum along the magnetic field, and a discrete parameter $n$ related to the finite dynamics in the plane orthogonal to the magnetic field. The number of states for the lowest Landau level is different from the others; Landau degeneracy factor for the lowest level is $|e B| / 2 \pi$ while for the other is $|e B| / \pi$. Our purpose will be to show that the dynamics of the lowest Landau level (LLL) is the one playing crucial role in the chiral symmetry breaking.

The dynamics of the chiral condensates in an external magnetic field has many interesting and important features. To make conclusions for those which we will use in the next sections, let us start with expressing the chiral condensate through the fermion propagator $S(x, y)$

$$
\langle 0|\bar{\Psi} \Psi| 0\rangle=\lim _{x \rightarrow y} \operatorname{tr} S(x, y)
$$

Thus, the problem we are going to study is encoded in the properties, or more concretely the pole structure of the fermion propagator $S(x, y)$.

The fermion propagator is well known form a long time and is usually defined as the matrix element

$$
\begin{aligned}
S(x, y) & =\left(i \gamma^{\mu} D_{\mu}^{x}+m\right)\left\langle x\left|\frac{-i}{\left(\gamma^{\mu} D_{\mu}\right)^{2}+m^{2}}\right| y\right\rangle \\
& =\left(i \gamma^{\mu} D_{\mu}^{x}+m\right) \int_{0}^{\infty} d s\left\langle x\left|e^{-i s\left[\left(\gamma^{\mu} D_{\mu}\right)^{2}+m^{2}\right]}\right| y\right\rangle .
\end{aligned}
$$

To calculate the matrix element $\left\langle x\left|\exp \left(-i s\left[\left(\gamma^{\mu} D_{\mu}\right)^{2}+m^{2}\right]\right)\right| y\right\rangle$, one can use the Schwinger's proper time approach, which gives

$$
\left\langle x\left|e^{-i s\left[\left(\gamma^{\mu} D_{\mu}\right)^{2}+m^{2}\right]}\right| y\right\rangle=\frac{e^{-i(\pi / 4)}}{8(\pi s)^{3 / 2}} e^{i\left[S_{c l}-s m^{2}\right]}\left(e B s \cot (e B s)+\gamma^{1} \gamma^{2} e B s\right) .
$$

In the above expression, $S_{c l}$ is defined as

$$
S_{c l}=e \int_{y}^{x} A_{\nu}^{e x t} d x^{\nu}-\frac{1}{4 s}(x-y)_{\nu}\left[g^{\nu \mu}+\frac{\left(\left(F^{e x t}\right)^{2}\right)_{\nu \mu}}{B^{2}}(1-e B s \cot (e B s))\right](x-y)_{\mu^{\prime}}
$$

where the integration is along a straight line connecting the two points since the result is path independent. 
Separating the phase factor containing the integration, the propagator can be represented in the following convenient form:

$$
S(x, y)=e^{i e \int_{y}^{x} A_{v}^{e x t} d x^{v}} \tilde{S}(x-y)
$$

where

$$
\begin{aligned}
\tilde{S}(x)=-i & \int_{0}^{\infty} \frac{d s}{16(\pi s)^{2}} e^{-i s m^{2}} e^{-(i / 4 s)\left[\left(x^{0}\right)^{2}-x_{A}^{2}(e B s) \cot (e B s)-\left(x^{3}\right)^{2}\right]} \\
& \cdot\left(m+\frac{1}{2 s}\left[\gamma^{0} x^{0}-\gamma^{A} x_{A}(e B s) \cot (e B s)-\gamma^{3} x^{3}\right]-\frac{e B}{2} \epsilon_{A B} \gamma^{A} x^{B}\right) \\
& \cdot\left((e B s) \cot (e B s)-\gamma^{2} \gamma^{2}(e B s)\right), \quad A=1,2, \quad \epsilon_{12}=+1 .
\end{aligned}
$$

It is more convenient to consider the propagator in the Euclidean momentum space. Transforming to the Euclidean momentum space $\left(k^{0} \rightarrow i k_{4}, s \rightarrow-i s\right)$, we get

$$
\begin{aligned}
\langle 0|\bar{\Psi} \Psi| 0\rangle & =\frac{-i}{(2 \pi)^{2}} \operatorname{tr} \int d^{4} k \widetilde{S}_{E}(k) \\
& =\frac{4 m}{(2 \pi)^{2}} \int d^{4} k \int_{1 / \Lambda}^{\infty} d s e^{-s\left(m^{2}+k_{4}^{2}+k_{3}^{2}+k_{A}^{2}(\tanh (e B s) / e B s)\right)} \\
& =\frac{e B m}{(2 \pi)^{2}} \int_{1 / \Lambda}^{\infty} \frac{d s}{s} e^{-s m^{2}} \operatorname{coth}(e B s)
\end{aligned}
$$

where $\Lambda$ is UV cutoff. It is instructive to look at the behavior of the condensate for the infinitesimal $m$. The last expression in this limit takes the form

$$
\langle 0|\bar{\Psi} \Psi| 0\rangle \stackrel{m \rightarrow 0}{\longrightarrow}-|e B s| \frac{m}{4 \pi^{2}}\left(\log \frac{\Lambda^{2}}{m^{2}}+\mathcal{O}\left(m^{0}\right)\right) .
$$

It is clear that the logarithmic singularity is due to the contributions from large distances, that is, for large proper time $s$. The conclusion one can draw about the role of the magnetic field is that it confines effectively the dynamics in only two dimensions, that is, we arrive at $1+1$ dynamical problem. To uncover the nature of the logarithmic singularity, let us take a closer look at the fermion propagator in Euclidean signature

$$
\begin{aligned}
\widehat{S}_{E}(k)= & -i \int_{0}^{\infty} d s e^{-\left(m^{2}+k_{4}^{2}+k_{3}^{2}\right) s} e^{-\left(k_{\perp}^{2} / e B\right) \tanh (e B s)}\left(1+\frac{1}{i} \gamma_{1} \gamma_{2} \tanh (e B s)\right) \\
& \cdot\left(-k_{\mu}+m+\frac{1}{i} \gamma_{A} k_{B} \epsilon^{A B} \tanh (e B s)\right) .
\end{aligned}
$$


It is obvious that all the terms can be obtained by differentiating on parameters or integrating by parts of the expression

$$
I=\int_{0}^{\infty} d s e^{-\tilde{\rho} s} e^{-(\lambda / 2) \tanh (e B s)},
$$

where $\lambda=k_{\perp}^{2} / e B, \tilde{\rho}=m^{2}+k_{3}^{2}+k_{4}^{2}$. The second exponent can be expanded over Laguerre polynomials $L_{n}^{\alpha}$ using the generating function $(z=-\exp (-2 e B s))$

$$
e^{\lambda / 2} e^{(\lambda / 2) \cdot((z-1) /(z+1))}=\sum_{n=0}^{\infty} c_{n}(\lambda) z^{n}, \quad c_{n}(\lambda)=L_{n}(\lambda)-L_{n-1}(\lambda), \quad|z|<1 .
$$

The final expression one can obtain after lengthy but straightforward calculations is

$$
\widehat{S}_{E}(k)=-i e^{-k_{\perp}^{2} / e B} \sum_{n=0}^{\infty}(-1)^{n} \frac{D_{n}(e B, k)}{k_{4}^{2}+k_{3}^{2}+m^{2}+2 e B n},
$$

where

$$
\begin{aligned}
D_{n}(e B, k)= & 4\left(k_{1} \gamma_{1}+k_{2} \gamma_{2}\right) L_{n}^{1}\left(2 \frac{k_{\perp}^{2}}{e B}\right) \\
& +\left(m-k_{4} \gamma_{4}-k_{3} \gamma_{3}\right)\left[\left(1-i \gamma_{1} \gamma_{2}\right) L_{n}\left(2 \frac{k_{\perp}^{2}}{e B}\right)-\left(1+i \gamma_{1} \gamma_{2}\right) L_{n-1}\left(2 \frac{k_{\perp}^{2}}{e B}\right)\right] .
\end{aligned}
$$

Thus, the poles of the propagator are located at the Landau levels! From this result, one can draw the following important conclusions. Analyzing the terms in the propagator, one can see that the logarithmic singularity in the condensate is due to the lowest Landau level. The second conclusion is that the above expression explicitly shows the $1+1$ nature of the lowest Landau level dynamics. Thus, the dynamics of the fermion pairing in a magnetic field in $4 \mathrm{~d}$ is $1+1$ dimensional phenomenon.

Summarizing, we stress on the conclusion that the presence of a magnetic field drives spontaneous chiral symmetry breaking even when the field strength is weak. The mechanism is fairly universal since it catalyzes the fermion pairing at the lowest Landau level. The pairing dynamics is essentially $1+1$ dimensional in the infrared region. Concluding this section, we note that the generation of dynamical masses can be illustrated on the examples of concrete models described in the literature (see e.g., $[5,18,19])$.

\section{Holographic Flavor Dynamics in a Nutshell}

The idea of gauge/string duality is one of the most profound in the realm of fundamental interactions. It influenced a lot both sides of the correspondence: since the first papers on the subject appear, several new important ideas and results have emerged in string and gauge theories. 
A crucial milestone was the large $N$ limit proposed by $t^{\prime}$ Hooft [20]. Instead of using $S U(3)$ as a gauge group, 't Hooft proposed to consider $S U(N)$ Yang-Mills theory and take the limit $N \gg 1$, while keeping the so-called 't Hooft coupling fixed $\lambda=N g_{Y M}^{2}$. 't Hooft proved that in this limit only planar diagrams contribute to the partition function which makes the theory more tractable. On the other side, the expansion in $1 / N$ corrections of the QCD partition function and the genus expansion of the string partition function exhibit the same qualitative behavior, suggesting that perhaps a dual description of the large $N$ limit of non-abelian gauge theories might be attainable in the framework of string theory.

In this section we will discuss some qualitative and quantitative aspects of the holographic correspondence between strings and gauge theories. Once the correspondence is argued, our primary interest will be focused on the introduction of favors and their dynamics.

\subsection{The AdS/CFT Correspondence}

Let us make the above ideas more concrete introducing the basic ingredients of the socalled AdS/CFT correspondence. In our outline, we will assume basic knowledge of the concepts of superstring theory and the notion of D-branes. (We refer the reader to [21] for a comprehensive introduction to the subject.)

One can conceive of two basic types of strings. The first are the so-called closed strings, which at any moment of time have the topology $S^{1}$. It turns out that the closed strings define a consistent perturbation theory in and of themselves, and that it is this case that leads to the type II supergravities the equation of motion. One might also consider the socalled open strings which, at any instant of time, have the topology of an interval. In order for the dynamics of such strings to be well defined, one must specify boundary conditions at the ends. One possibility is to impose Neumannn boundary conditions to describe the free ends. Another possibility is to impose Dirichlet boundary conditions requiring the end points of the string to remain fixed at some points of the spacetime. One can also consider a mixture of Dirichlet and Neumann boundary conditions, insisting that the end of the string remain, attached to some submanifold of spacetime, but otherwise leaving it free to roam around the surface. Surfaces associated with such Dirichlet boundary conditions are known as Dirichlet submanifolds, that is, D-branes. To shorten the long discussion, we just stress that it turns out that the Dirichlet submanifolds are sources of the Ramond-Ramond gauge fields and of the gravitational field. That is, they carry both stress-energy and Ramond-Ramond charges. Summarizing, one can say that in many ways, the discovery of D-branes was a breakthrough for string theory. D-branes provide nonperturbative solutions to the theory. They also couple naturally to both open strings, which have gauge fields in their spectrum, and to closed strings, which have gravitons as vibration modes. This complementary nature of D-branes makes for a powerful framework for further study of the ideas of gauge/string duality.

The idea of the gauge/string duality passed though many controversial developments over few decades. The early hints about a possible gauge/string duality came very close to reality with the development of the concept of Dp-branes and their identification as the sources of the well-known black p-brane solutions of type IIB supergravity. The key observation was that the low energy dynamics of a stack of $N$ coincident Dpbranes can be equally well described by an $S U(N)$ supersymmetric Yang-Mills theory in $p+1$ dimensions and an appropriate limit of a p-brane gravitational background. 
The first gauge theory studied in this context is the large $N \mathcal{N}=4 S U(N)$ supersymmetric Yang-Mills theory in $1+3$ dimensions which is a maximally supersymmetric conformal field theory. The corresponding gravitational background is, as proposed by Maldacena [1, 2], the near horizon limit of the extremal 3-brane solution of type IIB supergravity. This was the original formulation of the standard (by now) AdS/CFT correspondence.

An important step was the understanding of the role of one of the additional coordinates (supplementing the four usual ones) as a renormalization-group scale. The idea was further promoted demonstrating the possibility of self-consistent account of the back reaction of the gravity on the D-branes and vice versa. Further development of this idea leads to the formulation of the AdS/CFT correspondence. We refer the reader to the extensive review [22] for a detailed historical overview and a detailed list of references and focus on the physical aspects of the correspondence.

\section{Low Energy Dynamics of D3-Branes and the Decoupling Limit}

When there are $N$ parallel Dp-branes, their low energy dynamics is described by a reduction of the $N=1$ supersymmetric ten-dimensional Yang-Mills theory of the gauge group $U(N)$ to the $p+1$ dimensional supersymmetric Yang-Mills-Higgs theory. The physics of the supersymmetric Yang-Mills systems can be understood by that of the D-brane dynamics and vice versa.

Let us consider a stack of $N$ coincident D3-branes. This system has two different kinds of perturbative-type IIB string theory excitations, namely, open strings that begin and end on the stack of branes and closed strings which are the excitations of empty space. Let us focus on the low-energy massless sector of the theory.

The first type of excitations corresponds to zero length strings that begin and end on the D3-branes. The orientation of these strings is determined by the D3-brane that they start from and the D3-brane that they end on. Thus, the states describing the spectrum of such strings are labeled by $\lambda_{i j}$, where $i, j=1, \ldots, N$. It can be shown that in the case of oriented strings [21] $\lambda_{i j}$ transform in the adjoint representation of $U(N)$. On the other side, the massless spectrum of the theory should form an $N=4$ supermultiplet in $1+3$ dimensions. The possible form of the interacting theory (if we take into account only interactions among the open strings) is thus completely fixed by the large amount of supersymmetry that we have and is the $N=4 U(N)$ supersymmetric Yang-Mills theory. Note that $U(N)$ came from the transformation properties of $\lambda_{i j}$. On the other side, $U(N)$ can be thought of as a direct product of $U(1)$ and $S U(N)$, geometrically the $U(1)$ corresponds to the collective coordinates of the stack of D3-branes. We will restrict ourselves to the case when those modes were not excited; we refer the reader to [22] for further discussion on this point.

The second kind of excitations is that of type IIB closed strings in flat space. The lowenergy massless sector is thus a type IIB supergravity in $1+9$ dimensions.

The complete action of the system is a sum of the actions of those two different sectors plus an additional interaction term. This term can be arrived at by covariantizing the brane action after introducing the background metric for the brane [23]. It can be shown [22] that in the $\alpha^{\prime} \rightarrow 0$ limit the interaction term vanishes and the two sectors of the theory decouple.

To summarize, the low energy massless perturbative excitations of the stack of D3branes are given by two decoupled sectors, namely, $N=4 S U(N)$ supersymmetric Yang-Mills theory and supergravity in flat $1+9$ space-time. Our next step is to consider an equivalent description of this system in terms of effective supergravity solution. 
Let us consider the extremal black 3-brane solution of type IIB supergravity. The corresponding gravitational background is given by [21]

$$
\begin{gathered}
d s^{2}=H_{3}^{-1 / 2} \eta_{\mu \nu} d x^{\mu} d x^{\nu}+H_{3}^{1 / 2} d x^{i} d x^{i}, \\
e^{2 \Phi}=g_{s}^{2} \\
C_{(4)}=H_{3}^{-1} g_{s}^{-1} d x^{0} \wedge \cdots \wedge d x^{3},
\end{gathered}
$$

where $\mu=0, \ldots, 3, i=4, \ldots, 9$, and the harmonic (in six dimensions) function $H_{3}$ is given by

$$
H_{3}=1+\frac{4 \pi g_{3} N \alpha^{\prime 2}}{r^{4}}
$$

The integer number $N$ quantizes the flux of the five-form field strength $d C_{(4)}$. It can also be interpreted as the number of D3-branes sourcing the geometry. We note that the elementary D3-brane solutions for small $y$ have a warp factor describing a "throat" geometry. For very large $y$, the throat opens into a flat $\mathbb{R}^{1,4}$ space. Taking the near horizon limit of the geometry corresponds to sending $\alpha^{\prime} \rightarrow 0$ while keeping the quantity $u=r / \alpha^{\prime}$ fixed. Such a limit serves two goals: first it enables one to zoom in the geometry near the extremal horizon, and second it corresponds to a low energy limit in the string theory defined on this background. After leaving only the leading terms in $\alpha^{\prime}$, one can obtain the following metric $[1,2]$ :

$$
\begin{gathered}
d s^{2}=\frac{u^{2}}{R^{2}}\left(-d x_{0}^{2}+d x_{1}^{2}+d x_{2}^{2}+d x_{3}^{2}\right)+R^{2} \frac{d u^{2}}{u^{2}}+R^{2} d \Omega_{5}^{2} \\
C_{(4)}=\frac{1}{g_{s}} \frac{u^{4}}{R^{4}} d x^{0} \wedge d x^{1} \wedge d x^{2} \wedge d x^{3} \\
e^{\Phi}=g_{s}, \\
R^{4}=4 \pi g_{s} N_{c} \alpha^{\prime 2} .
\end{gathered}
$$

The background in (3.3) is that of an $\mathrm{AdS}_{5} \times S^{5}$ space-time of radius $R$. Note that from a point of view of an observer at $r \rightarrow \infty$, the type IIB string theory excitations living in the near horizon area, namely, superstring theory on the background (3.3), will be redshifted by an infinite factor of $\sqrt{g_{t t}}=H_{3}^{-1 / 4}$. Therefore, we conclude that type IIB superstring theory on the background of $\mathrm{AdS}_{5} \times S^{5}$ should contribute to the low-energy massless spectrum of the theory seen by an observer at infinity. However, an observer at infinity has another type of low-energy massless excitations of type IIB string theory, namely, type IIB supergravity on flat $1+9$ space or gravitational waves. Those two different types of excitations can be shown to decouple form each other. To verify this one can consider the scattering amplitudes of incident gravitons of the core of the geometry (the near horizon area). It can be shown that at low energies $(\omega \ll 1 / R)$ the absorption cross-section of such a scattering $\sigma_{\mathrm{abs}}$ goes like $[24,25] \sigma_{\mathrm{abs}} \propto \omega^{3} R^{8}$. Therefore, one has that $\sigma_{\mathrm{abs}} \rightarrow 0$ and those two types of excitations decouple in the low energy limit $\omega \rightarrow 0$.

Let us see what the decoupling limit means for the string sigma model in the D3-brane background. We will concentrate here on the metric part, thereby ignoring the contributions 
from the five-form field $F_{5}$. We denote the $D=10$ coordinates by $x^{M}, M=0,1, \ldots, 9$, and the metric by $G_{M N}(x)$. We choose the first 4 coordinates to coincide with $x^{\mu}$ of the Poincare invariant D3 worldvolume, while the coordinates on the 5-sphere are $x^{M}$ for $M=5, \ldots, 9$ and the coordinate $x^{4}=u$. The full D3-brane metric takes the form $d s^{2}=G_{M N} d x^{M} d x^{N}=$ $R^{2} \widetilde{G}_{M N}(x ; R) d x^{M} d x^{N}$, where the rescaled metric $\widetilde{G}_{M N}$ is given by

$$
\tilde{G}_{M N}(x ; R) d x^{M} d x^{N}=\left(1+\frac{R^{4}}{u^{4}}\right)^{1 / 2}\left(\frac{d u^{2}}{u^{2}}+d \Omega_{5}^{2}\right)\left(1+\frac{R^{4}}{u^{4}}\right)^{-1 / 2} \frac{\eta_{i j}}{u^{2}} d x^{i} d x^{\mathrm{j}}
$$

Substituting this metric back into the nonlinear sigma model, we obtain

$$
S_{G}=\frac{1}{4 \pi \alpha^{\prime}} \int_{\Sigma} \sqrt{-\gamma} \gamma^{m n} G_{M N}(x) \partial_{m} x^{M} \partial_{n} x^{N}=\frac{R^{2}}{4 \pi \alpha^{\prime}} \int_{\Sigma} \sqrt{-\gamma} \gamma^{m n} \tilde{G}_{M N}(x) \partial_{m} x^{M} \partial_{n} x^{N}
$$

The overall coupling constant for the sigma model dynamics is given by

$$
\frac{R^{2}}{4 \pi \alpha^{\prime}}=\sqrt{\frac{\lambda}{4 \pi}}, \quad \lambda \equiv g_{s} N
$$

Keeping $g_{s}$ and $N$ fixed but letting $\alpha^{\prime} \rightarrow 0$ implies that $R \rightarrow 0$. It is easy to see that under this limit the sigma model action admits a smooth limit, given by

$$
S_{G}=\sqrt{\frac{\lambda}{4 \pi}} \int_{\Sigma} \sqrt{-\gamma} \gamma^{m n} \tilde{\mathrm{G}}_{M N}(x) \partial_{m} x^{M} \partial_{n} x^{N},
$$

where the metric $\widetilde{G}_{M N}(x ; 0)$ is the metric on $\mathrm{AdS}_{5} \times S^{5}$,

$$
G_{M N}(x ; R) d x^{M} d x^{N}=\frac{\eta_{i j}}{u^{2}} d x^{i} d x^{j}+\frac{d u^{2}}{u^{2}}+d \Omega_{5}^{2}
$$

rescaled to unit radius. Moreover, the coupling $1 / \sqrt{\lambda}$ has taken over the role of $\alpha^{\prime}$ as the nonlinear sigma model coupling constant and the radius $R$ has canceled out.

\section{The AdS/CFT Correspondence}

As we learned from the above, the massless sector of the low energy dynamics of $N$ coincident D3-branes allows two possible descriptions. Conjecturing that these descriptions are equivalent is the core of Maldacena's AdS/CFT correspondence [1, 2]. Notice that in both descriptions one part of the decoupled sectors is a type IIB supergravity in flat space. Thus, we are naturally led to the conclusion that type IIB superstring theory on the background of $A d S_{5} \times S^{5}$ background is dual to $\mathcal{N}=4 S U(N)$ supersymmetric Yang-Mills theory in $1+3$ dimensions. We have presented this statement in a diagrammatic way in Figure 1. 

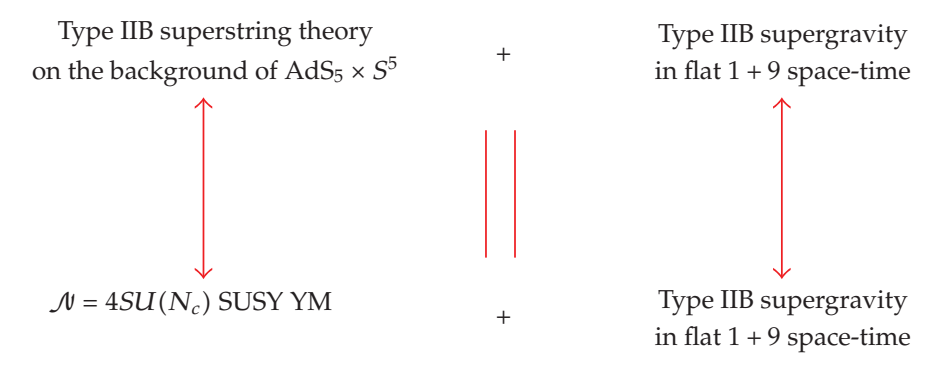

Figure 1: A diagrammatic statement of the Ads/Cft correspondence.

A further hint supporting the AdS/CFT correspondence is that the global symmetries of the proposed dual theories match. Indeed, an $\mathrm{AdS}_{5}$ space-time of radius $R$ can be embedded in an $\mathbb{R}^{2,6}$ flat space. It can then be naturally described as a hyperboloid of radius $R$ and thus has a group of isometry $S O(2,6)$ which is also the group of rotations in $2+4$ dimensions. On the other side, the $S^{5}$ part of the geometry has a group of isometry $S O(6)$ (the group of rotations in 6 dimensions). This leads us to the conclusion that the total global symmetry of string theory on $\mathrm{AdS}_{5} \times S^{5}$ gravitational background is $S O(2,4) \times S O(6)$. It is satisfying that the corresponding gauge theory has the same global symmetry. Indeed, it is a well-known fact that the $N=4$ supersymmetric YangMills theory in $1+3$ dimensions is a conformal field theory. As such it should has the global symmetry of the conformal group in $1+3$ dimensions, but this is precisely $S O(2,4)$. Actually, since the theory is supersymmetric, the full global symmetry group is the superconformal group which includes an $S U(4)$ global R-symmetry. In particular, this group rotates the gauginos of the super Yang-Mills theory. However, it is well known that $S U(4) \cong S O(6)$, and therefore the global symmetry of the gauge theory is indeed $S O(2,4) \times S O(6) !$

An important aspect of the correspondence is the regime of the validity of the dual description. Depending on the precise way in which we are taking the $\alpha^{\prime} \rightarrow 0$ limit, there are two basic forms of the AdS/CFT correspondence. The strongest form is that the string/gauge correspondence holds for any $N$. Unfortunately, this strong form of the conjecture cannot be tested directly since it is not known how to quantize superstring theory on a curved background in the presence of Ramond-Ramond fluxes [26]. The second form of the conjecture holds in the $t^{\prime}$ Hooft limit when $N \rightarrow \infty$ and the $t^{\prime}$ Hooft coupling $\lambda \propto g_{s} N$ is kept fixed. In this way on gauge side of the correspondence, only planar diagrams contribute to the partition function while on string side the required $g_{s} \rightarrow 0$ limit suggests semiclassical limit of the superstring theory on $\mathrm{AdS}_{5} \times S^{5}$. An important observation is that large $\lambda \gg 1$ suggests large AdS radius $R \propto \lambda^{1 / 4}$ and hence small curvature of the AdS background. This implies that the supergravity description is perturbative and thus provides an analytic tool for perturbative studies of nonperturbative field theory phenomena. On the other side, if we are at weak $t^{\prime}$ Hooft coupling $(\lambda \ll$ $1)$, we can perform perturbative studies on gauge side of the correspondence and transfer the result to the perturbatively inaccessible regime of the supergravity description. Therefore, we conclude that the AdS/CFT correspondence is a strong/weak duality. In this work, we will concentrate solely on the study of strongly coupled $\lambda \gg 1$ Yang-Mills theories. Hence, we will perform the analysis on the supergravity side of the AdS/CFT correspondence. 


\section{The AdS/CFT Dictionary}

It did not take long until an explicit formulation of Maldacenas conjecture was found. Gubser et al. [27] and Witten [28] independently proposed to identify the classical on-shell supergravity action, expressed in terms of given boundary values, with the effective action of super Yang-Mills theory, where the supergravity boundary values play the roles of generating currents. Moreover, Witten suggested that via this identification any field theory action on $(d+1)$-dimensional anti-de Sitter space gives rise to an effective action of a field theory on the $d$-dimensional boundary of Anti-de Sitter space. Most importantly, this field theory on the boundary must be a conformal field theory, because the AdS symmetries act as conformal symmetries on the asymptotic boundary. This duality has since been called the AdS/CFT correspondence.

The general correspondence formula is [28]

$$
\int_{\Psi_{0}} D \Psi e^{-I_{\mathrm{AdS}}[\Psi]}=\left\langle\exp \int d^{d} x \mathcal{O}(x) \Psi_{0}(x)\right\rangle,
$$

where the functional integral on the left-hand side is over all fields $\Psi$ whose asymptotic boundary values are $\Psi_{0}$, and $\mathcal{O}$ denotes the conformal operators of the boundary conformal field theory.

In the classical limit, which will be considered exclusively throughout this Chapter, the functional integral on the left-hand side of (3.9) becomes redundant, and the correspondence formula can be given in the simple, form $[27,28]$ :

$$
I_{\mathrm{AdS}}\left[\Psi_{0}\right]=W_{\mathrm{CFT}}\left[\Psi_{0}\right]
$$

where $I_{\text {AdS }}$ is the classical on-shell action of an AdS field theory, expressed in terms of the field boundary values $\Psi_{0}$, and $W_{\text {CFT }}$ is the CFT effective action with generating currents, given by minus the logarithm of the right-hand side of (3.9). However, one must expect $I_{\mathrm{AdS}}$ to be divergent as it stands, because of the divergence of the AdS metric on the AdS horizon, $x_{0}=0$. Thus, in order to extract the physically relevant information, the on-shell action has to be renormalized by adding counter terms, which cancel the infinities. After defining the renormalized, finite action by

$$
I_{\mathrm{AdS}, \text { fin }}=I_{\mathrm{AdS}}-I_{\mathrm{div}}
$$

where $I_{\text {div }}$ stands for the local counter terms, one identifies $I_{\text {Ads,fin }}$ with the CFT effective action. Thus, the meaningful correspondence formula is

$$
I_{\mathrm{AdS}, \text { fin }} \equiv W_{\mathrm{CFT}} \text {. }
$$

Given a field theory action on AdS space and a suitable regularization method, it is straightforward to calculate the renormalized on-shell action $I_{\mathrm{AdS} \text {,fin }}$. On the other hand, the CFT effective action

$$
W_{\mathrm{CFT}}\left[\Psi_{0}\right]=-\ln \left\langle\exp \int d^{d} x \mathcal{O}(x) \Psi_{0}(x)\right\rangle
$$


contains all information about the conformal field theory living on the AdS horizon, in that all correlation functions of its operators can be obtained in a standard fashion. Thus, the AdS/CFT correspondence formula (3.12) provides for the most amazing fact that the properties of certain conformal field theories can be obtained from seemingly unrelated theories, namely, field theories on AdS spaces. Moreover, any field theory on AdS space, which includes gravity, has a corresponding counterpart CFT, whose action might not even be known. Thus, the AdS/CFT correspondence might be an invaluable tool for formulating nontrivial CFT's in various dimensions, although studies of this aspect.

Let us focus on the precise way that the AdS/CFT correspondence is implemented on the example of a scalar field $\phi$. After a closer look at the geometry of the $\mathrm{AdS}_{5} \times S^{5}$ background, we conclude that it has five noncompact directions. Four of them are parallel to the D3-branes world volume and correspond to the $1+3$ directions of the dual gauge theory. The fifth non-compact direction is the radial direction $u$ (radial in the transverse, to the D3-branes, $\mathbb{R}^{6}$ space) and its interpretation in the dual gauge theory is not obvious. To shed more light on it, let us consider the action of a free massless scalar field in $1+3$ dimensions [26]:

$$
S=\int d^{4} x(\partial \phi)^{2}
$$

The corresponding field theory is conformal and thus has a global symmetry $S O(2,4)$ which is the conformal group in $1+3$ dimensions. Therefore, we can consider the transformation properties of the scalar field $\phi$ under the action of the dilatation operator. One can verify that the transformation:

$$
x \longrightarrow e^{\alpha} x, \quad \phi \longrightarrow e^{-\alpha} \phi,
$$

leaves the action (3.14) invariant. Furthermore, we learn that the scalar field $\phi$ has a scaling dimension one. On the other side, the $S O(2,4)$ group is the group of isometry of $\mathrm{AdS}_{5}$ and one can verify from (3.3) that the transformation $x \rightarrow e^{\alpha} x$ suggests

$$
u \longrightarrow e^{-\alpha} u
$$

Therefore, we learn that the coordinate $u$ scales as energy under dilations and thus has a natural interpretation as an energy scale of the dual gauge theory.

The further development of the AdS/CFT correspondence resulted in a map between gauge invariant operators in $\mathcal{N}=4$ super Yang-Mills in a particular irreducible representation of the $S U(4)$ R-symmetry group and supergravity fields in the isomorphic representation of the $S O(6)$ global symmetry. These representations are obtained after Kaluza-Klein reduction of the supergravity fields on the internal $S^{5}$ sphere. Let us consider for simplicity the case of a scalar field of mass $m$, propagating on the $\mathrm{AdS}_{d+1}$ background. The relevant action is [26]

$$
S=\int d^{d} x d u \sqrt{-g}\left(g^{a b} \partial_{a} \phi \partial_{b} \phi-m^{2} \phi^{2}\right)
$$


The solution of the corresponding equation of motion the following asymptotic behavior at large $u$ :

$$
\phi(u, x)=\left(\frac{1}{u}\right)^{4-\Delta} \phi_{0}(x)+\left(\frac{1}{u}\right)^{\Delta}\langle\mathcal{O}(x)\rangle
$$

where

$$
\Delta=\frac{d}{2}+\sqrt{\frac{d^{2}}{4}+R^{2} m^{2}}
$$

Note that the supergravity field $\phi(u)$ is a scalar field and is thus invariant under the action of the dilatation operator because the latter is one of the generators of the global symmetry $S O(2,4)$. Therefore, we conclude that $\phi_{0}$ and $\langle\mathcal{O}(x)\rangle$ carry scaling dimensions $4-\Delta$ and $\Delta$, respectively. In [27] it was suggested that $\phi_{0}$ and $\langle\mathcal{O}(x)\rangle$ correspond to the source and the vacuum expectation value of the gauge invariant operator $\mathcal{O}(x)$. It is also worth noting that the expression:

$$
\int d^{d} x \phi_{0}(x)\langle\mathcal{O}(x)\rangle=\text { inv }
$$

is invariant under the $S O(2,4)$ global symmetry. It was suggested that the exact form of the map is given by the relation $[27,28]$ :

$$
\left\langle e^{\int d^{d} x \phi_{0}(x)\langle\mathcal{O}(x)\rangle}\right\rangle_{\mathrm{CFT}}=\mathfrak{Z}_{\text {Sugra }}\left[\phi_{0}(x)\right]
$$

where

$$
\mathfrak{Z}_{\text {Sugra }}\left[\phi_{0}(x)\right]=\lim _{\epsilon \rightarrow 0} \mathfrak{z}_{\text {Sugra }}\left[\phi_{0}\left(\frac{1}{\epsilon}, x\right)=\epsilon^{d-\Delta} \phi_{0}(x)\right] .
$$

That is, the generating functional of the conformal field theory coincides with the generating functional for tree level diagrams in supergravity. We refer the reader to the extensive review [22] for more subtleties on the precise way of taking the $\epsilon \rightarrow 0$ limit in (3.22).

Formula (3.21) has been tested by comparing correlation functions of the $N=4$ quantum field theory with classical correlation functions in $\mathrm{AdS}_{d}$. Note that the tree level approximation on supergravity side is valid only at strong 't Hooft coupling and therefore the corresponding conformal field theory is strongly coupled. This is why the correspondence was tested in this way only for correlation functions which satisfy non-renormalization theorems and hence are independent on the coupling [26]. In particular, it applies for the two- and three-point functions of $1 / 2$ BPS operators [29, 30].

Further checks of the correspondence beyond the $1 / 2$ BPS sector were started with the so-called plane-wave string/gauge theory duality, where one takes appropriate plane-wave limit of the $\mathrm{AdS}_{5} \times S^{5}$ background [31]. The key point of this limit is that superstring theory on this background can be exactly quantized. Recently a significant progress towards quantizing 
superstring theory on $\mathrm{AdS}_{5} \times S^{5}$ has been achieved using integrable spin chain models. We refer the reader to $[32,33]$ for more details on these vast subjects.

\subsection{Adding Flavors to the Correspondence}

Direct consequence of the confining property of QCD is the fact that the low energy dynamics of the theory is governed by color singlets, such as mesons, baryons, and glueballs. Mesons and baryons are bound states of quarks, the latter transform in the fundamental representation of $S U(3)$. The fact that at low energy QCD is strongly coupled suggests that it is not accessible for perturbative studies. This is why it is important to come up with an alternative nonperturbative techniques describing the strongly coupled regime of YangMills theories and in particular Yang-Mills theories containing matter in the fundamental representation of the gauge group, such as QCD.

Further need of alternative nonperturbative techniques applicable to the properties of the fundamental fields in the strongly coupled regime of non-abelian gauge theories is required by the very recent discoveries of the properties of matter obtained in heavy ion collision experiments. More precisely the fact is that the quark-gluon plasma, which is the phase of matter of the fireballs obtained in such experiments, is not the expected weakly coupled quark-gluon plasma predicted by the standard perturbative QCD but is classified as a strongly coupled quark-gluon plasma, a novel phase of matter that provides challenge for the society of theoretical physicists.

One of the purposes of the study of the AdS/CFT correspondence is to develop the above-mentioned analytic tools for the study of strongly coupled Yang-Mills theories. The original form of the conjecture that we described in the previous section focuses on a gauge theory with a huge amount of symmetry, namely, the $N=4$ super Yang-Mills theory. On way to make the correspondence more applicable to realistic gauge theories, such as QCD, is to reduce the amount of the supersymmetry of the theory by introducing additional gauge invariant operators. This approach though fruitful still has the weakness that the matter content of the dual gauge theory, more precisely the fermionic degrees of freedom, transforms in the adjoint representation of the gauge group. In other words, there are no fundamental fields in the theory. The reason is that both ends of the strings, producing the field content of the gauge theory, are attached to the same stack of D3-branes and the corresponding states transform in the adjoined representation of the gauge group. In order to introduce fundamental matter, one needs to consider separate stack of D-branes.

The easiest way to introduce fundamental fields in the context of the AdS/CFT correspondence is to consider an additional stack of $N_{f}$ D7-branes [3]. (From now on we will use $N_{c}$ as a notation for the number of the D3-branes sourcing the gravitational background.) Since the D7-branes' world volume is higher dimensional and non-compact in the transverse to the D3-branes dimensions, the D7-branes have infinite "internal" volume and thus their gauge coupling vanish making their gauge symmetry group a global symmetry. In this way, we introduce a family of fundamental matter with global flavor symmetry $\operatorname{SU}\left(N_{f}\right)$. To be more precise, let us consider two stacks of parallel $N_{c}$ D3-branes and $N_{f}$ D7-branes embedded in the following way (see Table 1).

The low energy spectrum of the strings stretched between the D3- and D7-branes directions gives rise to the $\mathcal{N}=2$ hypermultiplet containing two Weyl fermions of opposite chirality coming from the light-cone modes of strings stretched along the NN and DD directions $(2,3,8,9)$ and two complex scalars coming from strings stretched along the ND 
Table 1: Embedding of the flavor D7-branes.

\begin{tabular}{lllllllllll}
\hline & 0 & 1 & 2 & 3 & 4 & 5 & 6 & 7 & 8 & 9 \\
\hline D3 & - & - & $\cdot$ & $\cdot$ & $\cdot$ & $\cdot$ & $\cdot$ & $\cdot$ & $\cdot$ & $\cdot$ \\
D7 & - & - & - & - & $\cdot$ & $\cdot$ & - & - &. &. \\
\hline
\end{tabular}

directions, namely, 4,5,6,7. Now if we consider $N_{f} \ll N_{c}$ and take the large $N_{c}$ limit. We can substitute the stack of D3-branes with an $\mathrm{AdS}_{5} \times S^{5}$ space and study the $N_{f}$ D-branes in the probe limit using their Dirac-Born-Infeld action. On gauge side, this corresponds to working in the quenched approximation $\left(N_{f} \ll N_{c}\right)$ and taking the large $N_{c}^{\prime}$ t Hooft limit. If the D3- and D7-branes are separated in their transverse $(8,9)$-plane, then the strings stretched between them have a final length and hence final energy. It can be shown that [21] the mass of the hypermultiplet is given by the energy of the string or the distance of separation $L$ multiplied by the string tension $m_{q}=L /\left(2 \pi \alpha^{\prime}\right)$.

Let us study closer the symmetry of the set up. If the D3- and the D7-branes overlap the hypermultiplet is massless $\left(m_{q}=0\right)$. In this case, the $S O(6)$ rotational symmetry of the transverse $\mathbb{R}^{6}$ space is broken to the product $S O(4) \times S O(2)$, corresponding to rotations along the ND directions $(4,5,6,7)$ and the DD directions $(8,9)$, respectively. This is equivalent to an $S U(2)_{L} \times S U(2)_{R} \times U(1)_{R}$ global symmetry and suggests that the gauge theory has an Rsymmetry group $S O(2)_{R} \times U(1)_{R}$ [34], which is indeed the case, when the hypermultiplet is massless. If the D3- and D7-branes are separated, it is known that the R-symmetry is just $S U(2)_{R}$, which again fits the fact that the $S O(2)$ rotational symmetry in the $(8,9)$-plane is broken.

\section{The Dictionary of the Probe Brane}

Let us now focus on the precise way that the AdS/CFT dictionary is implemented. The dynamics of the D7-brane probe is described by the Dirac-Born-Infeld action including the Chern-Simons term [21]:

$$
\frac{S}{N_{f}}=-\mu_{7} \int_{\mathcal{M}_{8}} e^{-\Phi} d^{8} \xi \sqrt{-\operatorname{det}\left(P\left[G_{a b}\right]+\left(2 \pi \alpha^{\prime}\right)^{2} \mathcal{F}_{a b}\right)}+\frac{\left(2 \pi \alpha^{\prime}\right)^{2}}{2} \mu_{7} \int_{\mathcal{M}_{8}} P\left[C_{(4)}\right] \wedge \mathcal{F} \wedge \mathcal{F},
$$

where $\left(2 \pi \alpha^{\prime}\right) \mathcal{F}_{a b}=P\left[B_{a b}\right]+\left(2 \pi \alpha^{\prime}\right) F_{a b}$ and $\mu_{7}=\left[(2 \pi)^{7} \alpha^{\prime 4}\right]^{-1}$. It is convenient to introduce the following coordinates:

$$
\rho=u \cos \theta, \quad L=u \sin \theta,
$$

and consider the ansatz:

$$
L=L(\rho), \quad \phi=\text { const. }
$$


Then the lagrangian describing the D7-brane embedding is

$$
\mathcal{L} \propto \rho^{3} \sqrt{1+L^{\prime}(\rho)^{2}}
$$

leading to the equation of motion:

$$
L^{\prime}(\rho)=-\frac{2 c}{\sqrt{\rho^{6}-4 c^{2}}} .
$$

At large $\rho$ the solution has the following behavior:

$$
L(\rho)=m+\frac{c}{\rho^{2}}+\cdots
$$

Now if we introduce the field:

$$
\chi(u)=\frac{L(\rho)}{\rho^{2}+L(\rho)^{2}}=\frac{1}{u} m+\frac{1}{u^{3}} c+\cdots, \quad u^{2}=\rho^{2}+L(\rho)^{2},
$$

we can see that $\chi(u)$ has the same behavior as the field $\phi(u, x)$ from (3.18). This is quite suggesting. The asymptotic value of $L(\infty)=m$ is exactly the separation of the D3- and D7branes and is thus related to the mass of the hypermultiplet via $m_{q}=m /\left(2 \pi \alpha^{\prime}\right)$. Since the hypermultiplet chiral fields are our quarks, we will call $m_{q}$ the bare quark mass. Therefore, the coefficient $c$ should be proportional to the vev of the operator that couples to the bare quark mass but this is the quark condensate! This is an example of the how the generalized AdS/CFT dictionary works at the level of a D7-brane probing. Let us provide the exact relation between the quark condensate $\left\langle\mathcal{O}_{q}\right\rangle$ and the coefficient $c$ :

$$
\left\langle\mathcal{O}_{q}\right\rangle=-\frac{N_{f}}{\left(2 \pi \alpha^{\prime}\right)^{3} g_{Y M}^{2}} c .
$$

We refer the reader to the appendix of Chapter 2 for more details on the last calculation and to [35] for an elegant presentation of the holographic renormalization of probe D-branes in AdS/CFT.

Now let us go back to (3.27) and note that in order for the D7-brane to close smoothly in the bulk of the geometry, we need to impose $L^{\prime}(0)=0$. This is possible only for $c=$ 0 and thus we conclude that the condensate of the theory vanishs. But the dual gauge theory is supersymmetric this is why it is not surprising that the quark condensate is zero. Furthermore, since there is no potential between the D3- and D7-branes (because of the unbroken supersymmetry), the D7-brane should not bend at infinity and this is why the solution for the D7-brane embedding should be simply $L \equiv m$, as it is.

Note that the analysis that led to (3.30) requires that the gravitational background be only asymptotically $\mathrm{AdS}_{5} \times S^{5}$. In fact, in all cases that we are going to consider in this work, there will be some sort of the deformation of the bulk physics, coming either from 
the gravitational background or from the introduction of external fields. This will break the supersymmetry and will capacitate the dual gauge theory to develop a quark condensate. In Section 4, we will use this approach to provide a holographic description of magnetic catalysis of chiral symmetry breaking. Through the rest of this review, the study of the quark condensate as a function of the bare quark mass will enable us to explore the phase structure of the dual gauge theory and uncover a first-order phase transition associated to the melting of the light mesons of the theory.

\section{Magnetic Catalysis of Mass Generation in Holographic Gauge Theories}

The phenomenon of dynamical flavor symmetry breaking catalyzed by an arbitrarily weak magnetic field is known from $[5,18,19]$ and [6-8]. This effect was shown to be model independent and therefore insensitive to the microscopic physics underlying the low energy effective theory. In particular, the infra-red (IR) description of the Goldstone modes associated with the dynamically broken symmetry should be universal. One therefore expects to be able to study this phenomenon using the holographic formalism.

\subsection{Mass Generation in the D3/D7 Setup}

There are various ways in which one can study the breaking of the chiral symmetry holographically. This has been studied in the past by the deformation of $\mathrm{AdS}_{5} \times S^{5}$ by a field corresponding to a marginally irrelevant operator on the gauge theory side [36-38]. In the present case, however, we will stimulate the formation of a condensate by turning on the magnetic components of the $U(1)$ gauge field of the D7-branes $F_{\alpha \beta}$ (equivalent to exciting a pure gauge $B$-field in the supergravity background). This $U(1)$ gauge field corresponds to the diagonal $U(1)$ of the full $U\left(N_{f}\right)$ gauge symmetry of the stack of D7-branes. Since the D7-branes wrap an infinite internal volume, the dynamics of the $U\left(N_{f}\right)$ gauge field is frozen in the four-dimensional theory and the $U\left(N_{f}\right)$ gauge symmetry becomes a global flavor symmetry $U\left(N_{f}\right)=U(1)_{B} \times S U\left(N_{f}\right)$. Therefore, the $U(1)$ gauge field that we consider corresponds to the gauged $U(1)_{B}$ baryon symmetry and the magnetic field that we introduce couples to the baryon charge of the fundamental fields [39].

\subsubsection{Generalities}

The problem thus boils down to studying embeddings of probe D7-branes in the $\mathrm{AdS}_{5} \times S^{5}$ background parameterized as follows:

$$
\begin{gathered}
d s^{2}=\frac{\rho^{2}+L^{2}}{R^{2}}\left[-d x_{0}^{2}+d x_{1}^{2}+d x_{2}^{2}+d x_{3}^{2}\right]+\frac{R^{2}}{\rho^{2}+L^{2}}\left[d \rho^{2}+\rho^{2} d \Omega_{3}^{2}+d L^{2}+L^{2} d \phi^{2}\right], \\
d \Omega_{3}^{2}=d \psi^{2}+\cos ^{2} \psi d \beta^{2}+\sin ^{2} \psi d \gamma^{2}, \\
g_{s} C_{(4)}=\frac{u^{4}}{R^{4}} d x^{0} \wedge d x^{1} \wedge d x^{2} \wedge d x^{3}, \quad e^{\Phi}=g_{s}, \quad R^{4}=4 \pi g_{s} N_{c} \alpha^{\prime 2},
\end{gathered}
$$

where $\rho, \psi, \beta, \gamma$, and $L, \phi$ are polar coordinates in the transverse $\mathbb{R}^{4}$ and $\mathbb{R}^{2}$ planes, respectively. 
Here $x_{a=1 \cdots 3}, \rho, \psi, \beta, \gamma$ parameterize the world volume of the D7-brane and the following ansatz is considered for its embedding:

$$
\phi \equiv \text { const }, \quad L \equiv L(\rho)
$$

leading to the following induced metric on its worldvolume:

$$
d \widetilde{s}=\frac{\rho^{2}+L(\rho)^{2}}{R^{2}}\left[-d x_{0}^{2}+d x_{1}^{2}+d x_{2}^{2}+d x_{3}^{2}\right]+\frac{R^{2}}{\rho^{2}+L(\rho)^{2}}\left[\left(1+L^{\prime}(\rho)^{2}\right) d \rho^{2}+\rho^{2} d \Omega_{3}^{2}\right] .
$$

The D7-brane probe is described by the DBI action:

$$
S_{\mathrm{DBI}}=-N_{f} \mu_{7} \int_{\mathcal{M}_{8}} d^{8} \xi e^{-\Phi}\left[-\operatorname{det}\left(G_{a b}+B_{a b}+2 \pi \alpha^{\prime} F_{a b}\right)\right]^{1 / 2}
$$

Here $\mu_{7}=\left[(2 \pi)^{7} \alpha^{\prime 4}\right]^{-1}$ is the D7-brane tension, $G_{a b}$ and $B_{a b}$ are the induced metric and $B$-field on the D7-brane's world volume, while $F_{a b}$ is its worldvolume gauge field. A simple way to introduce a magnetic field is to consider a pure gauge $B$-field along the $x_{2}, x_{3}$ directions:

$$
B^{(2)}=H d x_{2} \wedge d x_{3}
$$

Since $B_{a b}$ and $F_{a b}$ appear on equal footing in the DBI action, the introduction of such a $B$-field is equivalent to introducing an external magnetic field of magnitude $H /\left(2 \pi \alpha^{\prime}\right)$ to the dual gauge theory.

Though the full solution of the embedding can only be calculated numerically, the large $\rho$ behaviour (equivalently the ultraviolet (UV) regime in the gauge theory language) can be extracted analytically:

$$
L(\rho)=m+\frac{c}{\rho^{2}}+\cdots
$$

As discussed in [37], the parameters $m$ (the asymptotic separation of the D7- and D3- branes) and $c$ (the degree of bending of the D7-brane in the large $\rho$ region) are related to the bare quark mass $m_{q}=m / 2 \pi \alpha^{\prime}$ and the fermionic condensate $\langle\bar{\psi} \psi\rangle \alpha-c$, respectively. It should be noted that the boundary behavior of $L(r)$ really plays the role of source and vacuum expectation value (vev) for the full $\mathcal{N}=2$ hypermultiplet of operators. In the present case, where supersymmetry is broken by the gauge field configuration, we are only interested in the fermionic bilinears and this will refer only to quarks, and not their supersymmetric counterparts.

At this point it is convenient to introduce dimensionless parameters $\tilde{c}=c / R^{3} H^{3 / 2}$ and $\tilde{m}=m / R \sqrt{H}$. By performing a numerical shooting method from the infrared while varying the small $\rho$ boundary value, $L(\rho \rightarrow 0)=L_{I R}$, we recover the parametric plot presented in Figure 2, the main result explored in [10]. 


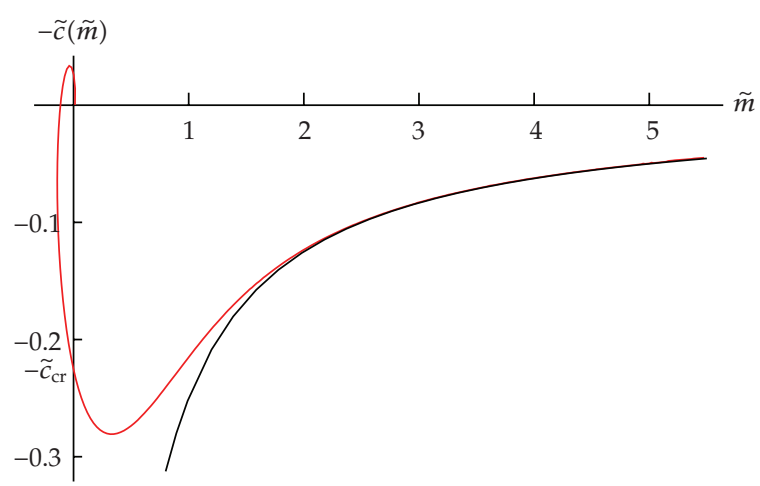

Figure 2: Parametric plot of $\tilde{c}$ against $\tilde{m}$ for fundamental matter in the presence of an external magnetic field. The lower (black) line represents the curve $1 / \tilde{m}$, fitting the large $\widetilde{m}$ behavior. It is also evident that for the outer branch of the spiral, for $\tilde{m}=0$ the condensate, $\langle\bar{\psi} \psi\rangle$ is non-zero. The corresponding value of the condensate is $\widetilde{c}_{\mathrm{cr}}=0.226$.

The lower (black) curve corresponds to the analytic behavior of $\tilde{c}(\tilde{m})=1 / \tilde{m}$ for large $\tilde{m}$. The most important observation is that at $\tilde{m}=0$ there is a non-zero fermionic condensate:

$$
\langle\bar{\psi} \psi\rangle=-\frac{N_{f} N_{c}}{\left(2 \pi \alpha^{\prime}\right)^{3} \lambda} c=-\frac{N_{f} N_{c} \widetilde{c}_{\mathrm{cr}}}{\left(2 \pi^{2}\right)^{3 / 4} \lambda^{1 / 4}}\left(\frac{H}{2 \pi \alpha^{\prime}}\right)^{3 / 2},
$$

Where $\lambda=g_{\mathrm{YM}}^{2} N_{c}$ is the $\mathrm{t}^{\prime}$ Hooft coupling and $\tilde{c}_{\mathrm{cr}} \approx 0.226$ is a numerical constant corresponding to the $y$-intercept of the outer spiral from Figure 2. Equation (4.7) is telling us that the theory has developed a negative condensate that scales as $\left(H / 2 \pi \alpha^{\prime}\right)^{3 / 2}$. This is not surprising, since the theory is conformal in the absence of the scale introduced by the external magnetic field. The energy scale controlled by the magnetic field, $\left(H / 2 \pi \alpha^{\prime}\right)^{1 / 2}$, leads to an energy density proportional to $\left(H / 2 \pi \alpha^{\prime}\right)^{2}$. In order to lower the energy, the theory responds to the magnetic field by developing a negative fermionic condensate.

Another interesting feature of the theory is the discrete-self-similar structure of the equation of state $(\tilde{c}$ versus $\tilde{m})$ in the vicinity of the trivial $\tilde{m}=0$ embedding, namely, the origin of the plot from Figure 2 presented in Figure 3.

This double logarithmic structure has been analyzed in [11], where a study of the meson spectrum revealed that only the outer branch of the spiral is tachyon free and corresponds to a stable phase having spontaneously broken chiral symmetry. In [12] it has been shown that an identical structure is also present for the D3/D5 system and it has been demonstrated that this structure is a universal feature of the magnetic catalysis of mass generation for gauge theories holographically dual to Dp/Dq intersections.

A further result of $[10,11,13]$ was the detailed analysis of the light meson spectrum of the theory. In [10] it was shown that the introduction of an external magnetic field breaks the degeneracy of the spectrum studied in [34]. This manifests itself as Zeeman splitting of the energy levels. In the limit of zero quark mass, the study also revealed the existence of a massless " $\eta$ ' meson" corresponding to the spontaneously broken $U(1)_{R}$ symmetry. In the next subsection, we will review the study of the meson spectrum of the theory. 


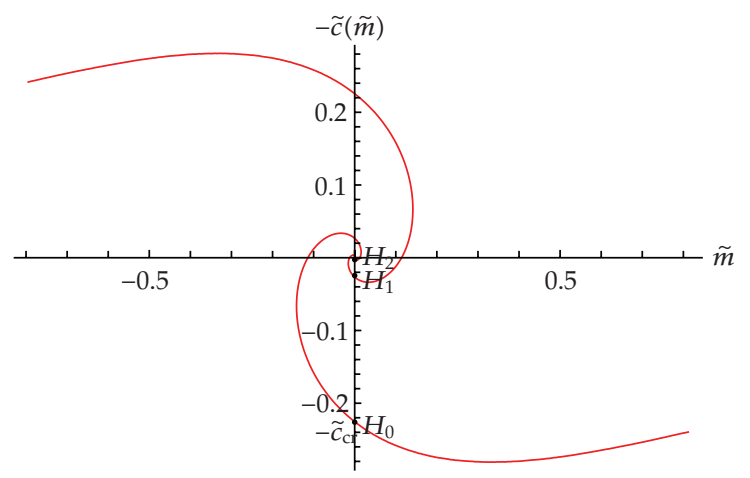

Figure 3: A magnification of Figure 2 shows the spiral behavior near the origin of the $(-\tilde{c}, \tilde{m})$-plane. The second (left) spiral arm represents the $(\tilde{m},-\widetilde{c}) \rightarrow(-\widetilde{m}, \widetilde{c})$ symmetry of the theory.

\subsubsection{Meson Spectrum}

\section{General Properties}

To study the scalar meson spectrum, one considers quadratic fluctuations [34] of the embedding of the D7-brane in the transverse $(L, \phi)$-plane. It can be shown that because of the diagonal form of the metric the fluctuation modes along the $\phi$ coordinate decouple from the one along $L$. However, because of the noncommutativity introduced by the $B$-field, we may expect the scalar fluctuations to couple to the vector fluctuations. This has been observed in [40], where the authors considered the geometric dual to noncommutative super Yang Mills as well as in the studies performed in $[10,11,13]$.

Let us proceed with obtaining the action for the fluctuations. To obtain the contribution from the DBI part of the action, we consider the following expansion:

$$
L=L_{0}(\rho)+2 \pi \alpha^{\prime} X, \quad \phi=0+2 \pi \alpha^{\prime},
$$

where $L_{0}(\rho)$ is the classical embedding of the D7-brane solution to the equation of motion derived from the action (4.4). To second order in $\alpha^{\prime}$, we have the following expression:

$$
E_{a b}=E_{a b}^{0}+2 \pi \alpha^{\prime} E_{a b}^{1}+\left(2 \pi \alpha^{\prime}\right)^{2} E_{a b^{\prime}}^{2}
$$

where $E^{0}, E^{1}, E^{2}$ are given by

$$
\begin{gathered}
E_{a b}^{0}=G_{a b}\left(\rho, L_{0}(\rho), \psi\right)+B_{a b}, \\
E_{a b}^{1}=\frac{R^{2} L_{0}^{\prime}}{\rho^{2}+L_{0}^{2}}\left(\partial_{a} X \delta_{b}^{\rho}+\partial_{b} X \delta_{a}^{\rho}\right)+\partial_{L_{0}} G_{a b} X+F_{a b} \\
E_{a b}^{2}=\frac{R^{2}}{\rho^{2}+L_{0}^{2}}\left(\partial_{a} X \partial_{b} X+L_{0}^{2} \partial_{a} \Phi \partial_{b} \Phi\right)-\frac{2 R^{2} L_{0} L_{0}^{\prime}}{\left(\rho^{2}+L_{0}^{2}\right)^{2}}\left(\partial_{a} X \delta_{b}^{\rho}+\partial_{b} X \delta_{a}^{\rho}\right) X+\frac{1}{2} \partial_{L_{0}}^{2} G_{a b} X^{2}
\end{gathered}
$$


Here $G_{a b}$ and $B_{a b}$ are the induced metric and $B$ field on the D7-brane's world volume. Now we can substitute (4.11) into (4.4)) and expand to second order in $\alpha^{\prime}$. It is convenient [40] to introduce the following matrices:

$$
\left\|E_{a b}^{0}\right\|^{-1}=S+J
$$

where $S$ is diagonal and $J$ is antisymmetric:

$$
\begin{gathered}
\left\|S^{a b}\right\|=\operatorname{diag}\left\{-G_{11}^{-1}, G_{11}^{-1}, \frac{G_{11}}{G_{11}^{2}+H^{2}}, \frac{G_{11}}{G_{11}^{2}+H^{2}}, G_{\rho \rho}^{-1}, G_{\psi \psi}^{-1}, G_{\alpha \alpha}^{-1}, G_{\beta \beta}^{-1}\right\}, \\
J^{a b}=\frac{H}{G_{11}^{2}+H^{2}}\left(\delta_{3}^{a} \delta_{2}^{b}-\delta_{3}^{b} \delta_{2}^{a}\right), \\
G_{11}=\frac{\rho^{2}+L_{0}^{2}}{R^{2}}, \quad G_{\rho \rho}=R^{2} \frac{\left(1+L_{0}^{\prime 2}\right)}{\rho^{2}+L_{0}^{2}}, \quad G_{\psi \psi}=\frac{R^{2} \rho^{2}}{\rho^{2}+L_{0}^{2}}, \\
G_{\alpha \alpha}=\cos ^{2} \psi G_{\psi \psi}, \quad G_{\beta \beta}=\sin ^{2} \psi G_{\psi \psi} .
\end{gathered}
$$

Now it is straightforward to get the effective action. At first order in $\alpha^{\prime}$, the action for the scalar fluctuations is the first variation of the classical action (4.4) and is satisfied by the classical equations of motion. Therefore, we focus on the second-order contribution from the DBI action.

After integrating by parts and taking advantage of the Bianchi identities for the gauge field, we end up with the following terms [10]. For $X$,

$$
\left\llcorner_{\chi} \propto \frac{1}{2} \sqrt{-E^{0}} \frac{R^{2}}{\rho^{2}+L_{0}^{2}} \frac{S^{a b}}{1+L_{0}^{\prime 2}} \partial_{a} \chi \partial_{b} X+\left[\partial_{L_{0}}^{2} \sqrt{-E^{0}}-\partial_{\rho}\left(\partial_{L_{0}} \sqrt{-E^{0}} \frac{L_{0}^{\prime}}{1+L_{0}^{\prime 2}}\right)\right] \frac{1}{2} \chi^{2},\right.
$$

and for $F$ :

$$
\mathcal{L}_{F} \propto \frac{1}{4} \sqrt{-E^{0}} S^{a a^{\prime}} S^{b b^{\prime}} F_{a b} F_{a^{\prime} b^{\prime}},
$$

and the mixed $x-F$ terms:

$$
\mathcal{L}_{F_{X}} \propto \frac{\sin 2 \psi}{2} f_{X} F_{23}
$$

and for $\Phi$,

$$
\mathcal{L}_{\Phi} \propto \frac{1}{2} \sqrt{-E^{0}} \frac{R^{2} L_{0}^{2}}{\rho^{2}+L_{0}^{2}} S^{a b} \partial_{a} \Phi \partial_{b} \Phi
$$


where the function $f$ in (4.15) is given by

$$
\begin{array}{r}
f(\rho)=\partial_{\rho}\left(g(\rho) \frac{L_{0}^{\prime}}{1+L_{0}^{\prime 2}} J^{23}\right)+J^{32} \partial_{L_{0}} g(\rho)+2 g(\rho) J^{23} S^{22} \partial_{L_{0}} G_{11} \\
\quad \text { with } \quad g(\rho)=\frac{\sqrt{-E^{0}}}{\sin \psi \cos \psi}=\rho^{3} \sqrt{1+L_{0}^{\prime 2}} \sqrt{1+\frac{R^{4} H^{2}}{\left(\rho^{2}+L_{0}^{2}\right)^{2}}} .
\end{array}
$$

As can be seen from (4.15), the $A_{2}, A_{3}$ components of the gauge field couple to the scalar field $\chi$ via the function $f$. Note that since for $\rho \rightarrow \infty$ and $L \rightarrow \infty$, we see that $J^{23} \rightarrow 0$, the mixing of the scalar and vector field decouples asymptotically. In order to proceed with the analysis we need, to take into account the contribution from the Wess-Zumino part of the action. The relevant terms to second order in $\alpha^{\prime}$ are [40]

$$
S_{W Z}=\frac{\left(2 \pi \alpha^{\prime}\right)^{2}}{2} \mu_{7} \int F_{(2)} \wedge F_{(2)} \wedge C_{(4)}+\left(2 \pi \alpha^{\prime}\right) \mu_{7} \int F_{(2)} \wedge B_{(2)} \wedge \tilde{P}\left[C_{(4)}\right]
$$

where $C_{(4)}$ is the background R-R potential given in (4.1) and $\widetilde{C}_{(4)}$ is the pull back of its magnetic dual. One can show that

$$
\widetilde{C}_{4}=-\frac{1}{g_{s}} \frac{R^{4} \rho^{4}}{\left(\rho^{2}+L^{2}\right)^{2}} \sin \psi \cos \psi d \psi \wedge d \alpha \wedge d \beta \wedge d \phi .
$$

Writing $\phi=2 \pi \alpha^{\prime} \Phi$, we write for the pull back $P\left[\widetilde{C}_{(4)}\right]$ :

$$
P\left[\widetilde{C}_{(4)}\right]=-\frac{2 \pi \alpha^{\prime}}{g_{s}} \frac{\sin 2 \psi}{2} K(\rho) \partial_{a} \Phi d \psi \wedge d \alpha \wedge d \beta \wedge d x^{a},
$$

where we have defined

$$
K(\rho)=\frac{R^{4} \rho^{4}}{\left(\rho^{2}+L_{0}^{2}\right)^{2}}
$$

Now note that the $B$-field has components only along $x^{2}$ and $x^{3}$; therefore, $d x^{a}$ in (4.20) can be only $d \rho, d x^{0}$, or $d x^{1}$. This will determine the components of the gauge field which can mix with $\Phi$. However, after integrating by parts and using the Bianchi identities, one can get the following simple expression for the mixing term:

$$
-\left(2 \pi \alpha^{\prime}\right)^{2} \frac{\mu_{7}}{g_{s}} \int d^{8} \xi \frac{\sin 2 \psi}{2} H \partial_{\rho} K \Phi F_{01}
$$


resulting in the following contribution to the complete lagrangian:

$$
\mathcal{L}_{F \Phi} \propto \frac{\sin 2 \psi}{2} H \partial_{\rho} K \Phi F_{01}
$$

Note that this means that only the $A_{0}$ and $A_{1}$ components of the gauge field couple to the scalar field $\Phi$. Next the contribution from the first term in (4.18) is given by

$$
\left(2 \pi \alpha^{\prime}\right)^{2} \frac{\mu_{7}}{g_{s}} \int d^{8} \xi \frac{\left(\rho^{2}+L_{0}^{2}\right)^{2}}{8 R^{4}} F_{a b} F_{c d} \epsilon^{a b c d}
$$

where the indices take values along the $\rho, \psi, \alpha, \beta$ directions of the world volume. This will contribute to the equation of motion for $A_{\rho}, A_{\psi}, A_{\alpha}$, and $A_{\beta}$ which does not couple to the scalar fluctuations. In this section, we will be interested in analyzing the spectrum of the scalar modes; therefore, we will not be interested in the components of the gauge field transverse to the D3-branes worldvolume. However, although there are no sources for these components from the scalar fluctuations, they still couple to the components along the D3branes as a result setting them to zero will impose constraints on the $A_{0} \cdots A_{3}$. Indeed, from the equation of motion for the gauge field along the transverse direction, one gets

$$
\sum_{a=0}^{3} S^{a a} \partial_{b} \partial_{a} A_{a}=0, \quad b=\rho, \psi, \alpha, \beta
$$

(Here, no summation on repeated indices is intended.) However, the nonzero $B$-field explicitly breaks the Lorentz symmetry along the D3-branes' world volume. In particular we have

$$
S^{00}=-S^{11}, \quad S^{22}=S^{33} \neq S^{11}
$$

which suggests that we should impose

$$
-\partial_{0} A_{0}+\partial_{1} A_{1}=0, \quad \partial_{2} A_{2}+\partial_{3} A_{3}=0
$$

We will see that these constraints are consistent with the equations of motion for $A_{0} \cdots A_{3}$. Indeed, with this constraint the equations of motion for $\chi, \Phi$ and $A_{\mu}, \mu=0 \cdots 3$ are, for $\chi$

$$
\begin{aligned}
& \frac{1+L_{0}^{\prime 2}}{g} \partial_{\rho}\left(\frac{g \partial_{\rho} X}{\left(1+L_{0}^{\prime 2}\right)^{2}}\right)+\frac{\Delta_{\Omega_{3}} X}{\rho^{2}}+\frac{R^{4}}{\left(\rho^{2}+L_{0}^{2}\right)^{2}} \widetilde{W}_{X} \\
& \quad+\frac{1+L_{0}^{\prime 2}}{g}\left(-\partial_{\rho}\left(\frac{\partial g}{\partial L_{0}} \frac{L_{0}^{\prime}}{1+L_{0}^{\prime 2}}\right)+\frac{\partial^{2} g}{\partial L_{0}^{2}}\right) x+\frac{1+L_{0}^{\prime 2}}{g} f F_{23}=0
\end{aligned}
$$


and for $\Phi$,

$$
\frac{1}{g} \partial_{\rho}\left(\frac{g L_{0}^{2} \partial_{\rho} \Phi}{1+L_{0}^{\prime 2}}\right)+\frac{L_{0}^{2} \Delta_{\Omega_{3}} \Phi}{\rho^{2}}+\frac{R^{4} L_{0}^{2}}{\left(\rho^{2}+L_{0}^{2}\right)^{2}} \widetilde{W} \Phi-\frac{H \partial_{\rho} K}{g} F_{01}=0
$$

and finally for $A_{a}$

$$
\begin{aligned}
& \frac{1}{g} \partial_{\rho}\left(\frac{g \partial_{\rho} A_{0}}{1+L_{0}^{\prime 2}}\right)+\frac{\Delta_{\Omega_{3}} A_{0}}{\rho^{2}}+\frac{R^{4}}{\left(\rho^{2}+L_{0}^{2}\right)^{2}} \widetilde{W} A_{0}+\frac{H \partial_{\rho} K}{g} \partial_{1} \Phi=0 \\
& \frac{1}{g} \partial_{\rho}\left(\frac{g \partial_{\rho} A_{1}}{1+L_{0}^{\prime 2}}\right)+\frac{\Delta_{\Omega_{3}} A_{1}}{\rho^{2}}+\frac{R^{4}}{\left(\rho^{2}+L_{0}^{2}\right)^{2}} \widetilde{W} A_{1}+\frac{H \partial_{\rho} K}{g} \partial_{0} \Phi=0 \\
& \frac{1}{g} \partial_{\rho}\left(\frac{g \partial_{\rho} A_{2}}{\left(1+L_{0}^{\prime 2}\right)\left(1+R^{4} H^{2} /\left(\rho^{2}+L_{0}^{2}\right)^{2}\right)}\right) \\
& \quad+\frac{R^{4}}{\left(\rho^{2}+L_{0}^{2}\right)^{2}+R^{4} H^{2}} \widetilde{W} A_{2}+\frac{\Delta_{\Omega_{3}} A_{2}}{\rho^{2}\left(1+R^{4} H^{2} /\left(\rho^{2}+L_{0}^{2}\right)^{2}\right)}-\frac{f}{g} \partial_{3} X=0, \\
& \frac{1}{g} \partial_{\rho}\left(\frac{g \partial_{\rho} A_{3}}{\left(1+L_{0}^{\prime 2}\right)\left(1+R^{4} H^{2} /\left(\rho^{2}+L_{0}^{2}\right)^{2}\right)}\right) \\
& \quad+\frac{R_{\Omega_{3}} A_{3}}{\left(\rho^{2}+L_{0}^{2}\right)^{2}+R^{4} H^{2}} \widetilde{W} A_{3}+\frac{\rho^{2}\left(1+R^{4} H^{2} /\left(\rho^{2}+L_{0}^{2}\right)^{2}\right)}{\left(\frac{f}{g} \partial_{2} X=0 .\right.}
\end{aligned}
$$

We have defined

$$
\widetilde{W}=-\partial_{0}^{2}+\partial_{1}^{2}+\frac{\partial_{2}^{2}+\partial_{3}^{2}}{1+R^{4} H^{2} /\left(\rho^{2}+L_{0}^{2}\right)^{2}} .
$$

As one can see the spectrum splits into two independent components, namely the vector modes $A_{0}, A_{1}$ couple to the scalar fluctuations along $\Phi$, while the vector modes $A_{2}, A_{3}$ couple to the scalar modes along $X$. However, it is possible to further simplify the equations of motion for the gauge field. Focusing on the equations of motion for $A_{0}$ and $A_{1}$ in equation (4.30), it is possible to rewrite them as

$$
\begin{gathered}
\frac{1}{g} \partial_{\rho}\left(\frac{g \partial_{\rho} F_{01}}{1+L_{0}^{\prime 2}}\right)+\frac{\Delta_{\Omega_{3}} F_{01}}{\rho^{2}}+\frac{R^{4}}{\left(\rho^{2}+L_{0}^{2}\right)^{2}} \widetilde{W} F_{01}-\frac{H \partial_{\rho} K}{g}\left(-\partial_{0}^{2}+\partial_{1}^{2}\right) \Phi=0 \\
\frac{1}{g} \partial_{\rho}\left(\frac{g \partial_{\rho}\left(-\partial_{0} A_{0}+\partial_{1} A_{1}\right)}{1+L_{0}^{\prime 2}}\right)+\frac{\Delta_{\Omega_{3}}\left(-\partial_{0} A_{0}+\partial_{1} A_{1}\right)}{\rho^{2}}+\frac{R^{4}}{\left(\rho^{2}+L_{0}^{2}\right)^{2}} \widetilde{W}\left(-\partial_{0} A_{0}+\partial_{1} A_{1}\right)=0 .
\end{gathered}
$$


Note that the first constraint in (4.27) trivially satisfies the second equation in (4.32). In this way we are left with the first equation in (4.32). Similarly, one can show that using the second constraint in (4.27) the equations of motion in (4.30) for $A_{2}$ and $A_{3}$ boil down to a single equation for $F_{23}$ :

$$
\begin{aligned}
& \frac{1}{g} \partial_{\rho}\left(\frac{g \partial_{\rho} F_{23}}{\left(1+L_{0}^{\prime 2}\right)\left(1+R^{4} H^{2} /\left(\rho^{2}+L_{0}^{2}\right)^{2}\right)}\right)+\frac{R^{4}}{\left(\rho^{2}+L_{0}^{2}\right)^{2}+R^{4} H^{2}} \widetilde{W} F_{23} \\
& \quad+\frac{\Delta_{\Omega_{3}} F_{23}}{\rho^{2}\left(1+R^{4} H^{2} /\left(\rho^{2}+L_{0}^{2}\right)^{2}\right)}+\frac{f}{g}\left(\partial_{2}^{2}+\partial_{3}^{2}\right) x=0 .
\end{aligned}
$$

Now let us proceed with a study of the fluctuations along $\Phi$.

\section{Fluctuations along $\Phi$ for a Weak Magnetic Field}

To proceed, we have to take into account the $F_{01}$ component of the gauge field strength and solve the coupled equations of motion. Since the classical solution for the embedding of the D7-brane is known only numerically, we have to rely again on numerics to study the meson spectrum. However, if we look at equation of motion derived from (4.4), we can see that the terms responsible for the non-trivial embedding of the D7-branes are of order $H^{2}$ [10]. On the other hand, the mixing of the scalar and vector modes due to the term (4.23) appears at first order in $H$. Therefore, it is possible to extract some non-trivial properties of the meson spectrum even at linear order in $H$ and as it turns out [10], we can observe a Zeeman-like effect: a splitting of states that is proportional to the magnitude of the magnetic field. Let us review the study performed in [10].

To first order in $H$ the classical solution for the D7-brane profile is given by

$$
L_{0}=m+O\left(H^{2}\right)
$$

where $m$ is the asymptotic separation of the D3- and D7-branes and corresponds to the bare quark mass. In this approximation, the expressions for $g(\rho)$ and $\partial_{\rho} K(\rho)$ become

$$
g(\rho)=\rho^{3}, \quad \partial_{\rho} K(\rho)=\frac{4 m^{2} R^{4} \rho^{3}}{\left(\rho^{2}+m^{2}\right)^{3}}
$$

and the equations of motion for $\Phi$ and $F_{01},(4.29)$ and (4.32), are simplified to

$$
\begin{aligned}
& \frac{1}{\rho^{3}}\left(\rho^{3} m^{2} \partial_{\rho} \Phi\right)+\frac{m^{2} \Delta_{\Omega_{3}}}{\rho^{2}} \Phi+\frac{m^{2} R^{4}}{\left(\rho^{2}+m^{2}\right)^{2}} \mathrm{~W} \Phi-4 H \frac{m^{2} R^{4}}{\left(\rho^{2}+m^{2}\right)^{3}} F_{01}=0 \\
& \frac{1}{\rho^{3}} \partial_{\rho}\left(\rho^{3} \partial_{\rho} F_{01}\right)+\frac{\Delta_{\Omega_{3}} F_{01}}{\rho^{2}}+\frac{R^{4}}{\left(\rho^{2}+m^{2}\right)^{2}} \mathrm{~W} F_{01}-4 H \frac{m^{2} R^{4}}{\left(\rho^{2}+m^{2}\right)^{3}} p^{2} \Phi=0
\end{aligned}
$$


where

$$
\mathrm{W}=-\partial_{0}^{2}+\partial_{1}^{2}+\partial_{2}^{2}+\partial_{3}^{2}, \quad p^{2}=-\partial_{0}^{2}+\partial_{1}^{2}
$$

This system has become similar to the system studied in [40] and in order to decouple it we can define the fields:

$$
\phi_{ \pm}=F_{01} \pm m P \Phi,
$$

where $p=\sqrt{-\partial_{0}^{2}+\partial_{1}^{2}}$. The resulting equations of motion are

$$
\frac{1}{\rho^{3}} \partial_{\rho}\left(\rho^{3} \partial_{\rho} \phi_{ \pm}\right)+\frac{\Delta_{\Omega_{3}}}{\rho^{2}} \phi_{ \pm}+\frac{R^{4}}{\left(\rho^{2}+m^{2}\right)^{2}} \mathrm{~W} \phi_{ \pm} \mp H \frac{4 R^{4} m}{\left(\rho^{2}+m^{2}\right)^{3}} p \phi_{ \pm}=0 .
$$

Note that $D^{2}$ is the Casimir operator in the $\left(x_{0}, x_{1}\right)$ plane only, while $\mathrm{W}$ is the Casimir operator along the D3-branes' world volume. If we consider a plane wave $e^{i x \cdot k}$, then we can define

$$
e^{i x \cdot k}=M^{2} e^{i x \cdot k}, \quad p^{2} e^{i x \cdot k}=M_{01}^{2} e^{i x \cdot k}
$$

and we have the relation:

$$
M^{2}=M_{01}^{2}-k_{2}^{2}-k_{3}^{2}
$$

The corresponding spectrum of $M^{2}$ is continuous in $k_{2}, k_{3}$. However, if we restrict ourselves to motion in the $\left(x_{0}, x_{1}\right)$-plane, the spectrum is discrete. Indeed, let us consider the following ansatz:

$$
\phi_{ \pm}=\eta_{ \pm}(\rho) e^{-i x_{0} k_{0}+i k_{1} x_{1}}
$$

Then we can write

$$
\frac{1}{\rho^{3}} \partial_{\rho}\left(\rho^{3} \partial_{\rho} \eta_{ \pm}\right)+\frac{R^{4}}{\left(\rho^{2}+m^{2}\right)^{2}} M_{ \pm}^{2} \eta_{ \pm} \mp H \frac{4 R^{4} m}{\left(\rho^{2}+m^{2}\right)^{3}} M_{ \pm} \eta_{ \pm}=0, \quad M_{ \pm} \equiv M_{01 \pm}
$$

Let us analyze (4.44). It is convenient to introduce

$$
\begin{gathered}
y=-\frac{\rho^{2}}{m^{2}}, \quad \bar{M}_{ \pm}=\frac{R^{2}}{m} M_{ \pm}, \quad P_{ \pm}(y)=(1-y)^{\alpha_{ \pm}} \eta_{ \pm \prime} \\
2 \alpha_{ \pm}=1+\sqrt{1+\bar{M}_{ \pm \prime}^{2}} \quad \epsilon=H \frac{R^{2}}{m^{2}} .
\end{gathered}
$$


With this change of variables, equation (4.44) is equivalent to

$$
y(1-y) P_{ \pm}^{\prime \prime}+2\left(1-\left(1-\alpha_{ \pm}\right) y\right) P^{\prime}-\alpha_{ \pm}\left(\alpha_{ \pm-1}\right) P_{ \pm} \pm \epsilon \frac{\bar{M}_{ \pm}}{(1-y)^{2}} P_{ \pm}=0
$$

Next we can expand

$$
\begin{aligned}
& P_{ \pm}=P_{0} \pm \epsilon P_{1}+O\left(\epsilon^{2}\right), \quad \alpha_{ \pm}=\alpha_{0} \pm \epsilon \alpha_{1}+O\left(\epsilon^{2}\right) \\
& \bar{M}_{ \pm}=\bar{M}_{0} \pm \epsilon \alpha_{1} \frac{\left(4 \alpha_{0}+2\right)}{\bar{M}_{0}}+O\left(\epsilon^{2}\right), \quad \bar{M}_{0}=2 \sqrt{\alpha_{0}\left(\alpha_{0}+1\right)}
\end{aligned}
$$

leading to the following equations for $P_{0}$ and $P_{1}$ :

$$
\begin{gathered}
y(1-y) P_{0}^{\prime \prime}+2\left(1-\left(1-\alpha_{0}\right) y\right) P_{0}^{\prime}-\alpha_{0}\left(\alpha_{0}-1\right) P_{0}=0 \\
y(1-y) P_{1}^{\prime \prime}+2\left(1-\left(1-\alpha_{0}\right) y\right) P_{1}^{\prime}-\alpha_{0}\left(\alpha_{0}-1\right) P_{1}=\left(\alpha_{1}\left(2 \alpha_{0}-1\right)-\frac{\bar{M}_{0}}{(1-y)^{2}}\right) P_{0}-2 \alpha_{1} y P_{0} .
\end{gathered}
$$

The first equation in (4.48) is the hypergeometric equation and corresponds to the fluctuations in pure $\mathrm{AdS}_{5} \times S^{5}$. It has the regular solution [34]:

$$
P_{0}(y)=F\left(-\alpha_{0}, 1-\alpha_{0}, 2, y\right)
$$

Furthermore, regularity of the solution for $\eta(\rho)$ at infinity requires [34] that $\alpha_{0}$ be discrete, and hence the spectrum of $\bar{M}_{0}$ :

$$
\begin{aligned}
& 1-\alpha_{0}=-n, \quad n=0,1, \ldots, \\
& \bar{M}_{0}=2 \sqrt{(n+1)(n+2)} .
\end{aligned}
$$

The second equation in (4.48) is an inhomogeneous hypergeometric equation. However, for the ground state, namely, $n=0, P_{0}=F(-1,0,2, y)=1$ and one can easily get the solution:

$$
P_{1}(y)=\frac{\bar{M}_{0}}{6} \ln (1-y)+\left(6 \alpha_{1}-\bar{M}_{0}\right)\left(\ln (-y)+\frac{1}{y}\right)-\frac{\bar{M}_{0}}{4(1-y)} .
$$

On the other hand, using the definition of $P_{ \pm}(y)$ in (4.45) to first order in $\epsilon$, we can write

$$
\eta_{ \pm}=\frac{1}{(1-y)^{\alpha_{0}}}\left(1 \mp \epsilon \frac{\alpha_{1}}{\alpha_{0}} \ln (1-y)\right)\left(1 \pm \epsilon P_{1}(y)\right)
$$




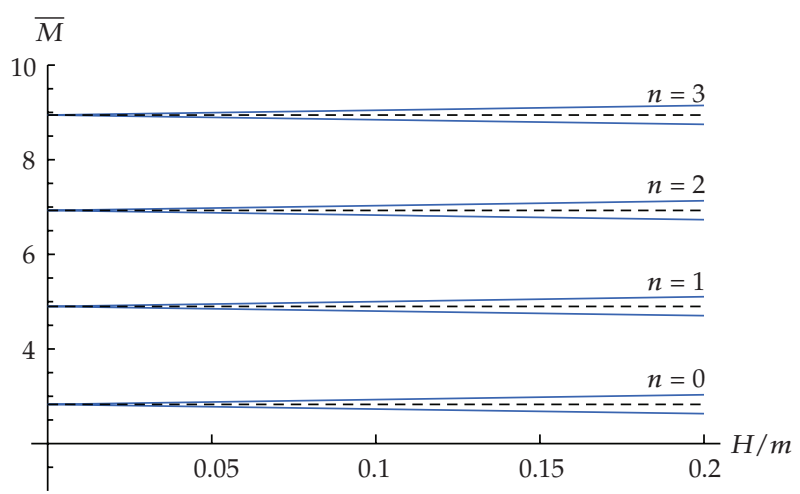

Figure 4: Plot of $\bar{M}=M R^{2} / m$ versus $H / m$ for the first three states. The dashed black lines correspond to the spectrum given by (4.50).

for the ground state $\alpha_{0}=1$ and we end up with the following expression for $\eta_{ \pm}$:

$$
\eta_{ \pm}=\frac{1}{1-y} \pm \epsilon \frac{\bar{M}_{0}}{4(1-y)^{2}} \pm \frac{\epsilon}{1-y}\left(6 \alpha_{1}-\bar{M}_{0}\right)\left(\ln (-y)+\frac{1}{y}-\frac{\ln (1-y)}{6}\right) .
$$

Now if we require that our solution is regular at $y=0$ and goes as $1 / \rho^{2} \propto 1 / y$ at infinity, the last term in (4.53) must vanish. Therefore, we have

$$
\alpha_{1}=\frac{\bar{M}_{0}}{6}
$$

After substituting in (4.47) and (4.45), we end up with the following correction to the ground sate [10]:

$$
M_{ \pm}=M_{0} \pm \frac{H}{m}
$$

We observe how the introduction of an external magnetic field breaks the degeneracy of the spectrum given by (4.50) and results in Zeeman splitting of the energy states, proportional to the magnitude of $H$. Although (4.55) was derived using the ground state, it is natural to expect that the same effect takes place for higher excited states. To demonstrate this, it is more convenient to employ numerical techniques for solving (4.44) and use the methods described in [36] to extract the spectrum. The resulting plot is presented in Figure 4. As expected, we observe Zeeman splitting of the higher excited states. It is interesting that (4.55) describes well not only the ground state, but also the first several excited states.

It turns out that one can easily generalize (4.55) to the case of non-zero momentum in the $\left(x_{2}, x_{3}\right)$-plane. Indeed, if we start from (4.40) and proceed with the following ansatz:

$$
\phi_{ \pm}=\tilde{\eta}_{ \pm}(\rho) e^{-i x \cdot k}
$$


we end up with

$$
\begin{gathered}
\frac{1}{\rho^{3}} \partial_{\rho}\left(\rho^{3} \partial_{\rho} \tilde{\eta}_{ \pm}\right)+\frac{R^{4}}{\left(\rho^{2}+m^{2}\right)^{2}} M_{ \pm}^{2} \widetilde{\eta}_{ \pm} \mp H \frac{4 R^{4} m}{\left(\rho^{2}+m^{2}\right)^{3}} M_{01 \pm} \tilde{\eta}_{ \pm}=0 \\
M_{01 \pm}=\sqrt{M_{ \pm}^{2}+k_{23}^{2}}, \quad k_{23} \equiv \sqrt{k_{2}^{2}+k_{3}^{2}}
\end{gathered}
$$

After going through the steps described in (4.45)-(4.53), (4.54) gets modified to

$$
\alpha_{1}=\frac{\bar{M}_{0}}{6} \sqrt{1+\frac{k_{23}^{2}}{M_{0}^{2}}}
$$

Note that validity of the perturbative analysis suggests that $\alpha_{1}$ is of the order of $\alpha_{0}$ and therefore we can trust the above expression as long as $k_{23}$ is of the order of $M_{0}$. Now it is straightforward to obtain the correction to the spectrum [10]:

$$
M_{ \pm}=M_{0} \pm \frac{H}{m} \sqrt{1+\frac{k_{23}^{2}}{M_{0}^{2}}}
$$

We see that the addition of momentum along the $\left(x_{2}-x_{3}\right)$-plane enhances the splitting of the states. Furthermore, the spectrum depends continuously on $k_{23}$.

\section{Fluctuations along $\Phi$ for a Strong Magnetic Field}

For strong magnetic field, we have to take into account terms of order $H^{2}$, which means that we no longer have an expression for $L_{0}(\rho)$ in a closed form and we have to rely on numerical calculations. We consider the following ansätz:

$$
\Phi=e^{i\left(k_{0} x^{0}+k_{1} x^{1}\right)} h(\rho), \quad F_{01}=e^{i\left(k_{0} x^{0}+k_{1} x^{1}\right)} f(\rho),
$$

and define

$$
M^{2}=k_{0}^{2}-k_{1}^{2}
$$

Equations (4.29) and (4.32) are simplified to

$$
\begin{aligned}
& \frac{1}{g} \partial_{\rho}\left(\frac{g L_{0}^{2}}{1+L_{0}^{\prime 2}} \partial_{\rho} h\right)+\frac{R^{4} L_{0}^{2}}{\left(\rho^{2}+L_{0}^{2}\right)^{2}} M^{2} h-\frac{H \partial_{\rho} K}{g} f=0, \\
& \frac{1}{g} \partial_{\rho}\left(\frac{g}{1+L_{0}^{\prime 2}} \partial_{\rho} f\right)+\frac{R^{4}}{\left(\rho^{2}+L_{0}^{2}\right)^{2}} M^{2} f-\frac{M^{2} H \partial_{\rho} K}{g} h=0 .
\end{aligned}
$$


Note that for large bare masses $m$ (and correspondingly large values of $L$ ), the term proportional to the magnetic field is suppressed and the meson spectrum should approximate to the result for the pure $\mathrm{AdS}_{5} \times S^{5}$ space-time case studied in [34], where the authors obtained the following relation:

$$
M_{n}=\frac{2 m}{R^{2}} \sqrt{(n+1)(n+3)}
$$

between the eigenvalue of the $n$th excited state $\omega_{n}$ and the bare mass $m$. If one imposes the boundary conditions:

$$
h(\epsilon)=1, \quad h^{\prime}(\epsilon)=0, \quad f(\epsilon)=1, \quad f^{\prime}(\epsilon)=0,
$$

the coupled system of differential equations can be solved numerically [12]. Then by requiring the functions $h(\rho)$ and $f(\rho)$ to be regular at infinity, one can quantize the spectrum of the fluctuations. It is also convenient to define the following dimensionless parameter $\widetilde{M}=M R / \sqrt{H}$. The resulting plot for the first three excited states is presented in Figure 5 [12]. There is Zeeman splitting of the states due to the magnetic field. (In the absence of the field, there are three straight lines emanating from the origin; these are split to form six curves.) Also, at zero bare quark mass, there is indeed a massless Goldstone mode, appearing at the end of the lowest curve. Furthermore, the plot in Figure 6 shows that for small bare quark mass one can observe a characteristic $\widetilde{M} \propto \sqrt{\tilde{m}}$ dependence. In the next section, we will review the analysis of the Goldstone mode performed in [12], where an analytic proof of the Gell-Mann-Oakes-Renner relation [9]:

$$
M_{\pi}^{2}=-\frac{2\langle\bar{\psi} \psi\rangle}{f_{\pi}^{2}} m_{q}
$$

in the spirit of [37] has been obtained.

Furthermore we will generalize the ansätz (4.60) to consider fluctuations depending on both the momentum along the magnetic field $\vec{k}_{\|}=\left(k_{1}, 0,0\right)$ and the transverse momentum $\vec{k}_{\perp}=\left(0, k_{2}, k_{3}\right)[12]:$

$$
\Phi=e^{i(\omega t+\vec{k} \cdot \vec{x})} h(\rho), \quad F_{01}=e^{i(\omega t+\vec{k} \cdot \vec{x})} f(\rho)
$$

We also review the result of [12] that for small $\omega=k_{0}$ and $|\vec{k}|$ the following dispersion relation holds:

$$
\omega(\vec{k})^{2}=M^{2}+\vec{k}_{\|}^{2}+\gamma \vec{k}_{\perp}^{2}, \quad \omega=k_{0} ; \vec{k}_{\|}=\left(k_{1}, 0,0\right), \vec{k}_{\perp}=\left(0, k_{2}, k_{3}\right),
$$

where $\gamma$ is a constant that has been determined. 


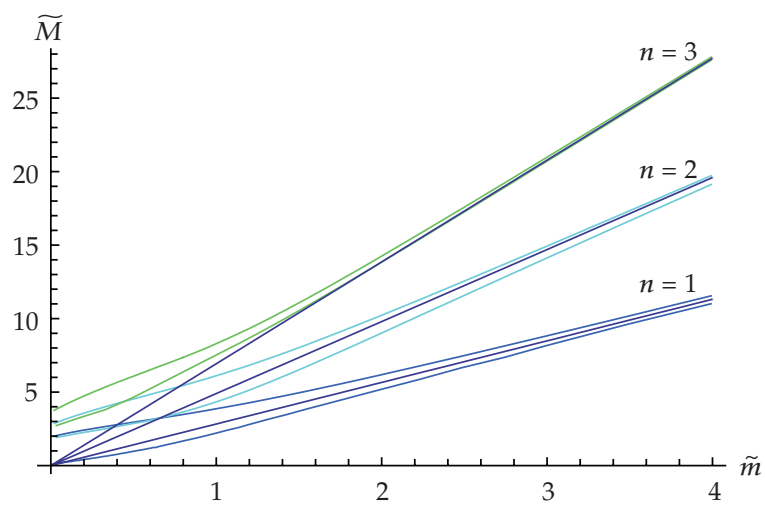

Figure 5: There is Zeeman splitting of the states due to the magnetic field. In the absence of the field, there are three straight lines emanating from the origin; these are split to form six curves. At zero bare quark mass (the end of the lowest curve), there is indeed a massless Goldstone mode. The straight lines correspond to the asymptotic AdS results.

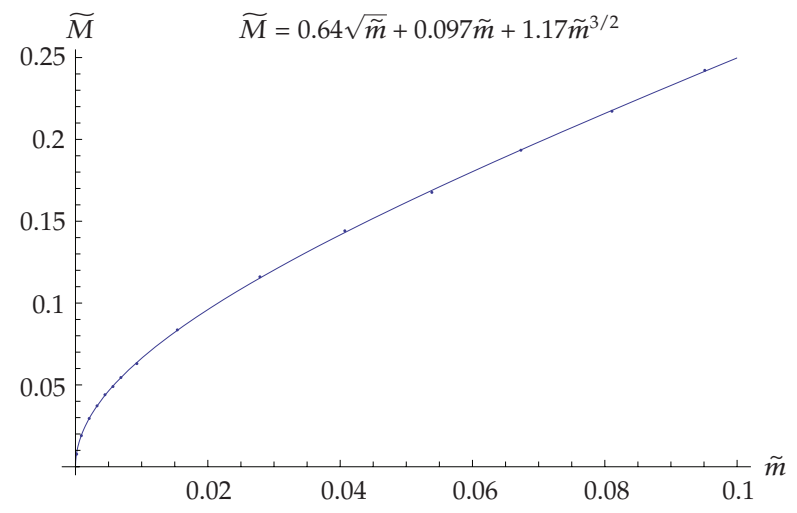

Figure 6: There is a characteristic $\widetilde{M} \propto \sqrt{\widetilde{m}}$ behavior at small bare quark mass.

\section{The $M^{2} \propto m$ Dependence}

Using an approach similar to the one employed in [37], the authors of [12] define

$$
\begin{gathered}
\Psi^{2}=\frac{g L_{0}^{2}}{1+L_{0}^{\prime 2}}, \quad v=R^{4} \frac{1+L_{0}^{\prime 2}}{\left(\rho^{2}+L_{0}^{2}\right)^{2}}, \quad \tilde{v}=R^{4} \frac{1+L_{0}^{\prime 2}}{\left(\rho^{2}+L_{0}^{2}\right)^{2}} \frac{1}{1+R^{4} H^{2} /\left(\rho^{2}+L_{0}^{2}\right)^{2}} \\
\Psi_{1}=\frac{\Psi}{L_{0}}, \quad \psi=h \Psi, \quad \psi_{1}=f \Psi_{1} .
\end{gathered}
$$


The equations of motions (4.29) and (4.32) can then be written in the following compact form:

$$
\begin{aligned}
& \ddot{\psi}-\frac{\ddot{\Psi}}{\Psi} \psi=-\left(\omega^{2}-\vec{k}_{\|}^{2}\right) v \psi+\vec{k}_{\perp}^{2} \tilde{\nu} \psi+\frac{H \partial_{\rho} K}{\Psi \Psi_{1}} \psi_{1} \\
& \ddot{\psi}_{1}-\frac{\ddot{\Psi}_{1}}{\Psi_{1}} \psi_{1}=-\left(\omega^{2}-\vec{k}_{\|}^{2}\right) v \psi_{1}+\vec{k}_{\perp}^{2} \tilde{\nu} \psi_{1}+\frac{H \partial_{\rho} K}{\Psi \Psi_{1}}\left(\omega^{2}-\vec{k}_{\|}^{2}\right) \psi .
\end{aligned}
$$

Let us remind the reader that for large $\rho, L_{0}(\rho)$ has the following behavior:

$$
L_{0} \propto m+\frac{c}{\rho^{2}}+\cdots
$$

Let us denote by $\bar{L}_{0}$ the classical embedding corresponding to $\left(m=0, c=c_{\mathrm{cr}}\right)$. It is relatively easy to verify that at $m=0, \vec{k}_{\perp}=\overrightarrow{0}$ and correspondingly $M^{2}=\omega^{2}-\vec{k}_{\|}^{2}=0$, the choice

$$
\psi=\left.\bar{\Psi} \equiv \Psi\right|_{\bar{L}_{0}}, \quad \psi_{1}=0,
$$

is a solution to the system (4.69). Next we consider embeddings corresponding to a small bare quark mass $\delta m$. This will correspond to small nonzero values of $M^{2}$ and $\vec{k}_{\perp}^{2}$. It is then natural to consider the following variations:

$$
\begin{aligned}
& \psi=\bar{\Psi}+\delta \psi, \\
& \psi_{1}=0+\delta \psi_{1},
\end{aligned}
$$

where $\delta \psi$ and $\delta \psi_{1}$ are of order $M^{2}$. Note that $M$ corresponds to the mass of the ground state at $m_{q}=\delta m / 2 \pi \alpha^{\prime}$ and we are assuming that the variations of the wave functions $\delta \psi$ and $\delta \psi_{1}$ are infinitesimal for infinitesimal $m_{q}$. After expanding in (4.69), we get the following equations of motion:

$$
\begin{gathered}
\delta \ddot{\psi}-\frac{\ddot{\bar{\Psi}}}{\bar{\Psi}} \delta \psi-\delta\left(\frac{\ddot{\Psi}}{\Psi}\right) \bar{\Psi}=-\left(\omega^{2}-\vec{k}_{\|}^{2}\right) \bar{\nu} \bar{\Psi}+\vec{k}_{\perp}^{2} \overline{\tilde{v}} \bar{\Psi}+\frac{H \partial_{\rho} K}{\bar{\Psi}_{1} \bar{\Psi}} \delta \psi_{1}, \\
\bar{\Psi}_{1} \delta \ddot{\psi}_{1}-\ddot{\bar{\Psi}}_{1} \delta \psi_{1}=H \partial_{\rho} K\left(\omega^{2}-k_{\|}^{2}\right),
\end{gathered}
$$

where $\bar{v}=\left.v\right|_{\bar{L}_{0}}$. The second equation in (4.73) can be integrated to give

$$
\bar{\Psi}_{1} \delta \dot{\psi}_{1}-\dot{\bar{\Psi}}_{1} \delta \psi_{1}=H K\left(\omega^{2}-k_{\|}^{2}\right)+\text { constant }
$$


From the boundary conditions that $\left.K\right|_{\rho=0}=0$ and $\bar{\Psi}_{1}(0)=0, \dot{\Psi}_{1}(0)=0$, we see that the constant of integration is zero and arrive at

$$
\partial_{\rho}\left(\frac{\delta \psi_{1}}{\bar{\Psi}_{1}}\right)=\frac{H K\left(\omega^{2}-k_{\|}^{2}\right)}{\bar{\Psi}_{1}^{2}}
$$

Next we multiply the first equation in (4.73) by $\bar{\Psi}$ and integrate along $\rho$ to obtain

$$
\begin{aligned}
\left(\omega^{2}-\vec{k}_{\|}^{2}\right) \int_{0}^{\infty} d \rho \overline{\mathcal{v}} \bar{\Psi}^{2}-\vec{k}_{\perp}^{2} \int_{0}^{\infty} d p \overline{\tilde{v}}^{2} \\
=-\int_{0}^{\infty}(\bar{\Psi} \delta \ddot{\psi}-\ddot{\bar{\Psi}} \delta \psi) d \rho+\int_{0}^{\infty} \bar{\Psi}^{2} \delta\left(\frac{\ddot{\Psi}}{\Psi}\right) d \rho+H \int_{0}^{\infty} \frac{\partial_{\rho} K \delta \psi_{1}}{\bar{\Psi}_{1}} d \rho \\
=-\left.(\bar{\Psi} \delta \dot{\psi}-\dot{\bar{\Psi}} \delta \psi)\right|_{0} ^{\infty}+\left.(\bar{\Psi} \delta \dot{\Psi}-\dot{\bar{\Psi}} \delta \Psi)\right|_{0} ^{\infty}-H \int_{0}^{\infty} K \partial_{\rho}\left(\frac{\delta \psi_{1}}{\bar{\Psi}_{1}}\right) d \rho,
\end{aligned}
$$

where the last term on the right-hand side of (4.76) has been integrated by parts using the fact that $\delta \psi_{1}$ should be regular at infinity. From the definition of $\bar{\Psi}$, it follows that $\bar{\Psi} \propto \rho^{3 / 2} L_{0}(0)$ as $\rho \rightarrow 0$ and $\bar{\Psi} \alpha c / \rho^{1 / 2}$ as $\rho \rightarrow \infty$. This, together with the requirement that $\psi_{1}$ is regular at $\rho=0$ and vanishes at infinity, suggests that the first term on the right-hand side of (4.76) vanishes. For the next term, we use the fact that

$$
\delta \Psi=\rho^{3 / 2} \delta\left(\frac{1+H^{2} R^{4} /\left(\rho^{2}+L_{0}^{2}\right)^{2}}{1+L_{0}^{\prime 2}}\right)^{1 / 4} L_{0}+\rho^{3 / 2}\left(\frac{1+H^{2} R^{4} /\left(\rho^{2}+L_{0}^{2}\right)^{2}}{1+L_{0}^{\prime 2}}\right)^{1 / 4} \delta L_{0}
$$

and therefore obtain

$$
\begin{gathered}
\left.\delta \Psi\right|_{0}=0,\left.\quad \delta \dot{\Psi}\right|_{0}=0, \\
\left.\delta \Psi\right|_{\infty} \propto \rho^{3 / 2} \delta m,\left.\quad \delta \dot{\Psi}\right|_{\infty} \propto \frac{3}{2} \sqrt{\rho} \delta m .
\end{gathered}
$$

The second term in (4.76) then becomes

$$
\left.(\bar{\Psi} \delta \dot{\Psi}-\dot{\bar{\Psi}} \delta \Psi)\right|_{0} ^{\infty}=2 c \delta m
$$

Finally using the equality in (4.75), we arrive at the following result:

$$
\left(\omega^{2}-\vec{k}_{\|}^{2}\right) \int_{0}^{\infty} d \rho\left\{\bar{v}^{2}+\frac{H^{2} \bar{K}^{2}}{\bar{\Psi}_{1}^{2}}\right\}-\vec{k}_{\perp}^{2} \int_{0}^{\infty} d \rho \overline{\widetilde{v}}^{2}=2 c \delta m
$$


Now we define [12]

$$
r=\frac{\left(\int_{0}^{\infty} d \rho \overline{\widetilde{v}} \bar{\Psi}^{2}\right)}{\left(\int_{0}^{\infty} d \rho\left\{\bar{v}^{2}+H^{2} \bar{K}^{2} / \bar{\Psi}_{1}^{2}\right\}\right)}
$$

and solve for $M^{2}$ from (4.67) to obtain

$$
M^{2} \int_{0}^{\infty} d \rho\left\{\bar{\nu} \bar{\Psi}^{2}+\frac{H^{2} \bar{K}^{2}}{\bar{\Psi}_{1}^{2}}\right\}=2 c \delta m .
$$

Equation (4.82) suggests that the mass of the "pion" associated to the softly broken global $U(1)$ symmetry satisfies the Gell-Mann-Oakes-Renner relation [9]:

$$
M_{\pi}^{2}=-\frac{2\langle\bar{\psi} \psi\rangle}{f_{\pi}^{2}} m_{q}
$$

In order to prove (4.83), we need to evaluate the effective coupling of the "pion" $f_{\pi}^{2}$. Noting that $\delta m \propto m_{q}$ and $c \alpha-\langle\bar{\psi} \psi\rangle$, we conclude that

$$
f_{\pi}^{2} \propto \int_{0}^{\infty} d \rho\left\{\bar{v} \bar{\Psi}^{2}+\frac{H^{2} \bar{K}^{2}}{\bar{\Psi}_{1}^{2}}\right\}
$$

To verify the consistency of their analysis, the authors of [12] compare the coefficient in (4.82) to the numerically determined coefficient 0.64 from the plot in Figure 6. Indeed from (4.82), it follows that

$$
\frac{\widetilde{M}}{\sqrt{\widetilde{m}}}=\left[\frac{1}{2 \widetilde{c}_{\mathrm{cr}}} \int_{0}^{\infty} d \tilde{\rho}\left\{\overline{\hat{v}} \overline{\widehat{\Psi}}^{2}+\frac{\overline{\widehat{K}}^{2}}{\overline{\widehat{\Psi}}_{1}^{2}}\right\}\right]^{-1 / 2} \approx 0.655
$$

where the dimensionless quantities:

$$
\widehat{v}=H^{2} v, \quad \widehat{\Psi}^{2}=\frac{\Psi^{2}}{R^{5} H^{5 / 2}}, \quad \widehat{\Psi}_{1}^{2}=\frac{\Psi_{1}^{2}}{R^{3} H^{3 / 2}}, \quad \widehat{K}=\frac{K}{R^{4}}
$$

have been defined. There is an excellent agreement with the fit from Figure 6.

Next we will obtain an effective four-dimensional action for the "pion" and from this derive an exact expression for $f_{\pi}^{2}$.

\section{Effective Chiral Action and $f_{\pi}^{2}$}

In this section, we will reduce the eight-dimensional worldvolume action for the quadratic fluctuations of the D7-brane to an effective action for the massless "pion" associated to the 
spontaneously broken global $U(1)$ symmetry. As rigid rotations along $\phi$ correspond to chiral rotations, (the asymptotic value of $\phi$ at infinity corresponds to the phase of the condensate in the dual gauge theory) the spectrum of $\phi$ at zero quark mass contains the Goldstone mode that we are interested in. This is why we first integrate out the gauge field components $A_{0}$ and $A_{1}$ and then dimensionally reduce to four dimensions [12].

Furthermore, as mentioned earlier, because of the magnetic field the $S O(1,3)$ Lorentz symmetry is broken down to $S O(1,1) \times S O(2)$ symmetry. This is why in order to extract the value of $f_{\pi}^{2}$ we consider excitations of $\Phi$ depending only on the $x_{0}, x_{1}$ directions and read off the coefficient in front of the kinetic term. The resulting on-shell effective action for $\Phi$ is [12]

$$
S^{\mathrm{eff}}=-\mathcal{N} \int d^{4} x\left[-\left(\partial_{0} \Phi\right)^{2}+\left(\partial_{1} \Phi\right)^{2}\right]
$$

where $\mathcal{N}$ is given by

$$
\mathcal{N}=\left(2 \pi \alpha^{\prime}\right)^{2} \frac{\mu_{7}}{g_{s}} N_{f} \pi^{2} \int_{0}^{\infty} d \rho\left\{\bar{\nu}^{2}+\frac{H^{2} \bar{K}^{2}}{\bar{\Psi}_{1}^{2}}\right\} .
$$

We refer the reader to the appendix of [12] for a detailed derivation of the $4 \mathrm{D}$ effective action $S^{\text {eff. }}$.

We have defined $\Phi$ via $\phi=\left(2 \pi \alpha^{\prime}\right) \Phi$, where $\phi$ corresponds to rotations in the transverse $\mathbb{R}^{2}$ plane and is the angle of chiral rotation in the dual gauge theory. The chiral Lagrangian is then given by

$$
S^{\mathrm{eff}}=-\left(2 \pi \alpha^{\prime}\right)^{2} \frac{f_{\pi}^{2}}{4} \int d^{4} x \partial_{\mu} \Phi \partial^{\mu} \Phi, \quad \mu=0 \text { or } 1,
$$

and therefore,

$$
f_{\pi}^{2}=N_{f} 4 \pi^{2} \frac{\mu_{7}}{g_{s}} \int_{0}^{\infty} d \rho\left(\bar{\nu} \bar{\Psi}^{2}+\frac{H^{2} K^{2}}{\bar{\Psi}_{1}^{2}}\right) .
$$

The D7-brane charge in (4.90) is given by $\mu_{7}=\left[(2 \pi)^{7} \alpha^{\prime^{4}}\right]^{-1}$ and the overall prefactor in (4.90) can be written as $N_{f} N_{c} / 2\left(2 \pi \alpha^{\prime}\right)^{4} \lambda$. Now, recalling the expressions for the fermionic condensate, (4.7), and the bare quark mass, $m_{q}=m / 2 \pi \alpha^{\prime}$, one can easily verify that (4.82) is indeed the Gell-Mann-Oakes-Renner relation:

$$
M_{\pi}^{2}=-\frac{2\langle\bar{\psi} \psi\rangle}{f_{\pi}^{2}} m_{q}
$$


It turns out that for small momenta $\vec{k}_{\|}, \vec{k}_{\perp}$ and small mass $M_{\pi}^{2}$, one can obtain the following more general effective $4 \mathrm{D}$ action (see Appendix A of [12] for a detailed derivation):

$$
S_{\text {eff }}=-\mathcal{N} \int d^{4} x\left\{\left[-\left(\partial_{0} \tilde{\Phi}\right)^{2}+\left(\partial_{1} \tilde{\Phi}\right)^{2}\right]+\gamma\left[\left(\partial_{2} \tilde{\Phi}\right)^{2}+\left(\partial_{3} \tilde{\Phi}\right)^{2}\right]-\frac{2\langle\bar{\psi} \psi\rangle}{f_{\pi}^{2}} m_{q} \widetilde{\Phi}^{2}\right\}+\cdots
$$

where $\gamma$ is defined in (4.81). As one can see, the action (4.92) is the most general quadratic action consistent with the $S O(1,1) \times S O(2)$ symmetry and suggests that pseudo-Goldstone bosons satisfy the dispersion relation (4.67).

\subsection{Mass Generation in the D3/D5 Setup}

In this section, we review the results of [12] providing a holographic description of the magnetic catalysis of chiral symmetry breaking in $1+3$-dimensional $S U\left(N_{c}\right) \mathcal{N}=4$ supersymmetric Yang-Mills theory coupled to $N_{f} \mathcal{N}=2$ fundamental hypermultiplets confined to a $1+2$-dimensional defect. In [12] it has been shown that the system develops a dynamically generated mass and negative fermionic condensate leading to a spontaneous breaking of a global $S O(3)$ symmetry down to a $U(1)$ symmetry. On the gravity side, this symmetry corresponds to the rotational symmetry in the transverse $\mathbb{R}^{3}$. The authors of [12] had shown that the $1+2$, dimensional nature of the defect theory leads to a coupling of the transverse scalars corresponding to the coset generators and as a result there is only a single Goldstone mode. It has also been shown that the characteristic $M_{\pi} \propto \sqrt{m}$ Gell-MannOakes-Renner relation is modified to a linear $M_{\pi} \propto m$ behavior. These features have been understood from a low-energy effective theory point of view. Indeed in $1+2$ dimensions the effect of the magnetic field is to break the $S O(1,2)$ Lorentz symmetry down to $S O(2)$ rotational symmetry and as a result the theory is nonrelativistic. A single time derivative chemical potential term is allowed. It is this term that is responsible for the modified counting rule of the number of Goldstone bosons [41] and leads to a quadratic dispersion relation as well as to the modified linear Gell-Mann-Oakes-Renner relation.

\subsubsection{Generalities}

Let us consider the $\mathrm{AdS}_{5} \times S^{5}$ supergravity background (4.1) and introduce the following parameterization:

$$
\begin{array}{r}
d s^{2}=\frac{u^{2}}{R^{2}}\left[-d x_{0}^{2}+d x_{1}^{2}+d x_{2}^{2}+d x_{3}^{2}\right]+\frac{R^{2}}{u^{2}}\left[d r^{2}+r^{2} d \Omega_{2}^{2}+d l^{2}+l^{2} d \widetilde{\Omega}_{2}^{2}\right] \\
u^{2}=r^{2}+l^{2}, \quad d \Omega_{2}^{2}=d \alpha^{2}+\cos ^{2} \alpha d \beta^{2}, \quad d \widetilde{\Omega}_{2}^{2}=d \psi^{2}+\cos ^{2} \psi d \phi^{2}
\end{array}
$$

We have split the transverse $\mathbb{R}^{6}$ to $\mathbb{R}^{3} \times \mathbb{R}^{3}$ and introduced spherical coordinates $r, \Omega_{2}$ and $l, \widetilde{\Omega}_{2}$ in the first and second $\mathbb{R}^{3}$ planes, respectively. Next we introduce a stack of probe $N_{f}$ D5-branes extended along the $x_{0}, x_{1}, x_{2}$ directions, and filling the $\mathbb{R}^{3}$ part of the geometry parameterized by $r, \Omega_{2}$. As mentioned above on the gauge theory side, this corresponds to introducing $N_{f}$ fundamental $\mathcal{N}=2$ hypermultiplets confined on a $1+2$ dimensional defect. 
The asymptotic separation of the D3-and D5 -branes in the transverse $\mathbb{R}^{3}$ space parameterized by $l$ corresponds to the mass of the hypermultiplet. In the following we will consider the following, ansätz for a single D5-brane:

$$
l=l(r), \quad \psi=0, \quad \phi=0 .
$$

The asymptotic separation $m=l(\infty)$ is related to the bare mass of the fundamental fields via $m_{q}=m / 2 \pi \alpha^{\prime}$. If the D3-and D5-branes overlap, the fundamental fields in the gauge theory are massless and the theory has a global $S O(3) \times S O(3)$ symmetry. Clearly a nontrivial profile of the D5-brane $l(r)$ in the transverse $\mathbb{R}^{3}$ would break the global symmetry down to $S O(3) \times U(1)$, where $U(1)$ is the little group in the transverse $\mathbb{R}^{3}$. If the asymptotic position of the D5-brane vanishes $(m=0)$, this would correspond to a spontaneous symmetry breaking, the non-zero separation $l(0)$ on the other hand would naturally be interpreted as the dynamically generated mass of the theory.

Note that the D3/D5 intersection is T-dual to the D3/D7 intersection from the previous section and thus the system is supersymmetric. The D3-and D5-branes are BPS objects and there is no attractive potential for the D5-brane, hence the D5-brane has a trivial profile $l \equiv$ const. However, a non-zero magnetic field will break the supersymmetry and as we are going to demonstrate, the D5-brane will feel an effective repulsive potential that will lead to dynamical mass generation. In order to introduce a magnetic field perpendicular to the plane of the defect, we consider a pure gauge $B$-field in the $x_{1}, x_{2}$ plane given by

$$
B=H d x_{1} \wedge d x_{2}
$$

This is equivalent to turning on a non-zero value for the 0,1 component of the gauge field on the D5-brane. The magnetic field introduced into the dual gauge theory in this way has a magnitude $H / 2 \pi \alpha^{\prime}$. The D5-brane embedding is determined by the DBI action:

$$
S_{\mathrm{DBI}}=-N_{f} \mu_{5} \int_{\mathcal{M}_{6}} d^{6} \xi e^{-\Phi}\left[-\operatorname{det}\left(G_{a b}+B_{a b}+2 \pi \alpha^{\prime} F_{a b}\right)\right]^{1 / 2},
$$

where $G_{a b}$ and $B_{a b}$ are the pull-back of the metric and the $B$-field, respectively, and $F_{a b}$ is the gauge field on the D5-brane.

With the ansätz (4.94), the lagrangian is given by

$$
\perp \propto r^{2} \sqrt{1+l^{\prime 2}} \sqrt{1+\frac{R^{4} H^{2}}{\left(r^{2}+l^{2}\right)^{2}}} .
$$

From this it is trivial to solve the equation of motion for $l(r)$ numerically, imposing $l(0)=l_{\text {in }}$ and $l^{\prime}(0)$ as initial conditions. Clearly, at large $r$ the lagrangian (4.97) asymptotes to that at zero magnetic field and hence we have the following asymptotic solution [42]:

$$
l(r)=m+\frac{c}{r}+\cdots,
$$

where $c \propto\langle\bar{\psi} \psi\rangle$ the condensate of the fundamental fields. 


\section{Spontaneous Symmetry Breaking}

Before solving the equation of motion, it is convenient to introduce dimensionless variables:

$$
\tilde{r}=\frac{r}{R \sqrt{H}}, \quad \tilde{l}=\frac{l}{R \sqrt{H}}, \quad \tilde{m}=\frac{m}{R \sqrt{H}}, \quad \tilde{c}=\frac{c}{R^{2} H}
$$

The lagrangian (4.97) can then be written as

$$
\perp \propto \tilde{r}^{2} \sqrt{1+\tilde{l}^{2}} \sqrt{1+\frac{1}{\left(\tilde{r}^{2}+\tilde{l}^{2}\right)^{2}}}
$$

The corresponding equation of motion is

$$
\partial_{\tilde{r}}\left(\frac{\tilde{r}^{2} l^{\prime}}{\sqrt{1+\tilde{l}^{2}}} \frac{\sqrt{1+\left(\tilde{r}^{2}+\tilde{l}^{2}\right)^{2}}}{\left(\tilde{r}^{2}+\tilde{l}^{2}\right)}\right)=-2 \frac{\tilde{r}^{2} \tilde{l} \sqrt{1+\tilde{l}^{2}}}{\left(\tilde{r}^{2}+\tilde{l}^{2}\right)^{2} \sqrt{1+\left(\tilde{r}^{2}+\tilde{l}^{2}\right)^{2}}} .
$$

Before solving (4.101), it will be useful to extract the asymptotic behavior of $\widetilde{c}(\tilde{m})$ at large $\tilde{m}$. To this end, we use that at large $\tilde{m}$ the separation $\tilde{l}(\tilde{r}) \approx \tilde{m}=$ const. The equation of motion then is simplified to

$$
\partial_{\tilde{r}}\left(\widetilde{r}^{2} \widetilde{l^{\prime}}\right)=-\frac{2 \widetilde{r}^{2} \tilde{m}}{\left(\widetilde{r}^{2}+\tilde{m}^{2}\right)^{3}}
$$

and hence

$$
\tilde{r}^{2} \tilde{l}^{\prime}=-2 \tilde{m} \int_{0}^{\tilde{r}} d \tilde{r} \frac{\tilde{r}^{2}}{\left(\tilde{r}^{2}+\tilde{m}^{2}\right)^{2}} .
$$

Using the expansion (4.98), one can verify that

$$
\lim _{\tilde{r} \rightarrow+\infty} \tilde{r}^{2} \widetilde{l^{\prime}}=\tilde{c}=2 \tilde{m} \int_{0}^{\infty} d \tilde{r} \frac{\tilde{r}^{2}}{\left(\tilde{r}^{2}+\tilde{m}^{2}\right)^{3}}=\frac{\pi}{8 \widetilde{m}^{2}}
$$

Equation (4.104) can thus be used as a check of the accuracy of our numerical results. Indeed the numerically generated plot of $-\tilde{c}$ versus $\tilde{m}$, is presented in Figure 7 . The most important observation is that at zero bare mass $\tilde{m}$ the theory has developed a negative condensate $\langle\bar{\psi} \psi\rangle \propto-\tilde{c}_{\mathrm{cr}} \approx-0.59$. It can also be seen that for large $\tilde{m}$ the numerically generated plot is in good agreement with (4.104) represented by the lower (black) curve. Another interesting feature of the equation of state is the spiral structure near the origin of the parameter space analogous to the one presented in Figure 3 for the case of the D3/D7 system. 


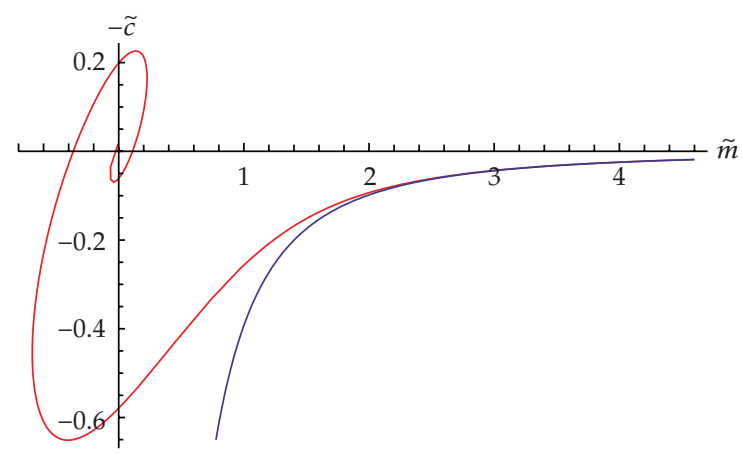

Figure 7: A plot of $-\tilde{c}$ versus $\tilde{m}$. At zero bare mass $\tilde{m}=0$, the theory has developed a negative condensate $\langle\bar{\psi} \psi\rangle \propto-\tilde{c}_{\mathrm{cr}} \approx-0.59$. For large $\tilde{m}$, there is an excellent agreement with (4.104), as represented by the lower (blue) curve.

In order to show that the global $S O(3)$ symmetry is indeed spontaneously broken we need to study the free energy of the theory. Indeed the existence of the spiral structure suggests that there is more than one phase at zero bare mass, corresponding to the different $y$-intercepts of the $-\tilde{c}$ versus $\tilde{m}$ plot. We will demonstrate below that the lowest positive branch of the curve presented in Figure 7 is the stable one.

Following [42] we will identify the regularized wick rotated on-shell action of the D5brane with the free energy of the theory. Let us introduce a cut-off at infinity, $r_{\max }$. The wick rotated on-shell action is given by

$$
S=N_{f} \frac{\mu_{5}}{g_{s}} 4 \pi V_{3} R^{3} H^{3 / 2} \int_{0}^{\tilde{r}_{\max }} d \tilde{r} \widetilde{r}^{2} \sqrt{1+\tilde{l}^{2}} \sqrt{1+\frac{1}{\left(\tilde{r}^{2}+\tilde{l}^{2}\right)^{2}}}
$$

where $V_{3}=\int d^{3} x$ and $\tilde{l}(\tilde{r})$ is the solution of (4.101). It is easy to verify, using the expansion from (4.98), that the integral in (4.105) has the following behavior at large $\widetilde{r}_{\max }$ :

$$
\int_{0}^{\tilde{r}_{\max }} d \tilde{r} \tilde{r}^{2} \sqrt{1+\tilde{l}^{2}} \sqrt{1+\frac{1}{\left(\tilde{r}^{2}+\tilde{l}^{2}\right)^{2}}}=\frac{1}{3} r_{\max }^{3}+O\left(\frac{1}{r_{\max }}\right) .
$$

It is important that in these coordinates the divergent term is independent of the field $\tilde{l}$; it is therefore possible to regularize the on-shell action by subtracting the free energy of the $\tilde{l} \equiv 0$ embedding. The resulting regularized expression for the free energy is

$$
F=S_{\text {reg }}=N_{f} \frac{\mu_{5}}{g_{s}} 4 \pi V_{3} R^{3} H^{3 / 2} \tilde{I}_{\mathrm{D} 5}
$$




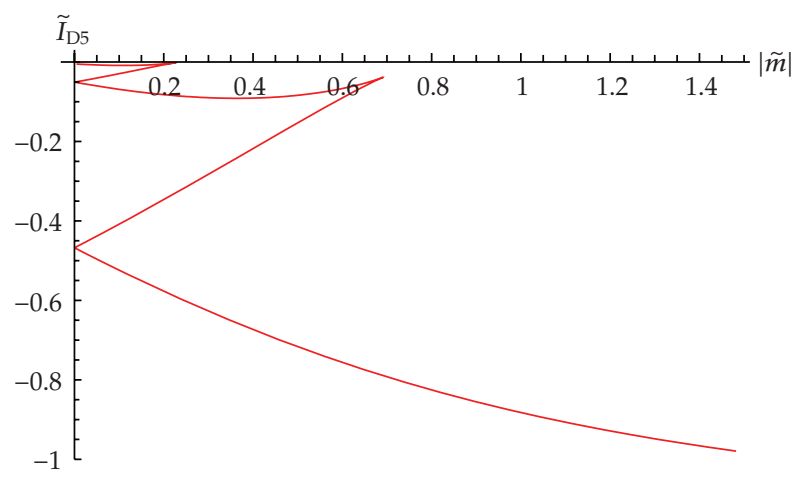

Figure 8: The states corresponding to the lowest positive branch of the plot in Figure 7 have the lowest free energy and thus correspond to the stable phase of the theory.

where

$$
\tilde{I}_{\mathrm{D} 5}=\int_{0}^{\infty} d \widetilde{r}\left[\tilde{r}^{2} \sqrt{1+\tilde{l}^{2}} \sqrt{1+\frac{1}{\left(\tilde{r}^{2}+\tilde{l}^{2}\right)^{2}}}-\sqrt{1+\tilde{r}^{4}}\right] .
$$

A plot of $\tilde{I}_{\mathrm{D} 5}$ versus $|\tilde{m}|$ is presented in Figure 8 . The states from the lowest positive branch in Figure 8 have the lowest free energy and correspond to the stable phase of the theory. Therefore, there is a spontaneous breaking of the global $S O(3)$ symmetry and the theory at $\tilde{m}=0$ develops a negative condensate proportional to $-\tilde{c}_{\mathrm{cr}} \approx-0.59$. Note that only the absolute value of $\tilde{m}$ corresponds to the bare mass of the fundamental fields. The states with negative $\tilde{m}$ correspond to D5-brane embeddings that intercept the $\tilde{l}=0$ line in the $\tilde{l}$ versus $\tilde{r}$ plane and as seen from Figure 8 are unstable. It is to be expected that the meson spectrum of the theory in such a phase would contain tachyons based on an analogy with the meson spectrum of the D3/D7 system studied in [11]. Before we proceed with the analysis of the meson spectrum of the theory, let us write an expression for the condensate of the theory $\langle\bar{\psi} \psi\rangle \propto-\mathcal{C}_{\mathrm{cr}}=R^{2} H \tilde{c}_{\mathrm{cr}}$ at zero bare quark mass. The coefficient of proportionality is given by [43]

$$
\langle\bar{\psi} \psi\rangle=-8 \pi^{2} \alpha^{\prime} \frac{\mu_{5}}{g_{s}} c_{\mathrm{cr}}=-16 \pi^{3} \alpha^{\prime 2} \frac{\mu_{5}}{g_{s}} \widetilde{c}_{\mathrm{cr}} R^{2}\left(\frac{H}{2 \pi \alpha^{\prime}}\right) .
$$

Note that the condensate is proportional to the magnitude of the magnetic field $H / 2 \pi \alpha^{\prime}$.

\subsubsection{Meson Spectrum and Pseudo-Goldstone Bosons}

In this section, we review the analysis of [12] on the normal modes of the D5-brane. The authors of [12] had focused on the normal modes corresponding to the Goldstone bosons of the spontaneously broken $S O(3)$ symmetry and studied their spectrum as a function of the bare quark mass $m_{q}$. The study shows that the external magnetic field splits the degeneracy of the meson spectrum and gives mass to one of the pions of the theory. It also modifies 
the standard $M_{\pi}^{2} \propto m$ GMOR relation for the remaining Goldstone mode to a linear relation $M_{\pi} \propto m$. It has been shown that these results are in accordance with the behavior expected from the effective chiral lagrangian of the theory.

In order to study the light meson spectrum of the theory, one looks for the quadratic fluctuations of the D5-brane embedding along the transverse directions parametrized by $l, \psi, \phi$. To this end, one expands

$$
l=\bar{l}+2 \pi \alpha^{\prime} \delta l, \quad \psi=2 \pi \alpha^{\prime} \delta \psi, \quad \phi=2 \pi \alpha^{\prime} \delta \phi
$$

in the action (4.96) and leaves only terms of order $\left(2 \pi \alpha^{\prime}\right)^{2}$. Note that fluctuations of the $U(1)$ gauge field $F_{\alpha \beta}$ of the D5-brane will also contribute to the expansion. There is also an additional contribution from the Wess-Zumino term of the D5-brane's action:

$$
S_{\mathrm{WZ}}=N_{f} \mu_{5} \int_{\mathcal{M}_{6}} \sum_{p}\left[C_{p} \wedge e^{\mp}\right] ; \quad \mathcal{F}=B+2 \pi \alpha^{\prime} F .
$$

The relevant term is

$$
S_{\mathrm{WZ}}=N_{f} \mu_{5} \int_{\mathcal{M}_{6}} B \wedge P\left[\tilde{C}_{4}\right]
$$

where $P\left[\widetilde{C}_{4}\right]$ is the pull-back of the magnetic dual, $\widetilde{C}_{4}$, to the background $C_{4} \mathrm{R}-\mathrm{R}$ form. It is given by

$$
\tilde{C}_{4}=\frac{1}{g_{s}} \frac{4 r^{2} l^{2}}{\left(r^{2}+l^{2}\right)^{3}} R^{4} \sin \psi(l d r-r d l) \wedge d \Omega_{2} \wedge d \phi
$$

The action for the quadratic fluctuations along $l$ is given by

$$
\begin{gathered}
\mathcal{L}_{l l}^{(2)} \propto \frac{1}{2} \sqrt{-E} G_{l l} \frac{S^{\alpha \beta}}{1+l^{\prime 2}} \partial_{\alpha} \delta l \partial_{\beta} \delta l+\frac{1}{2}\left[\partial_{l}^{2} \sqrt{-E}-\frac{d}{d r}\left(\frac{l^{\prime}}{1+l^{\prime 2}} \partial_{l} \sqrt{-E}\right)\right] \delta l^{2}, \\
\mathcal{L}_{l F}^{(2)} \propto \frac{\sqrt{-E}}{1+l^{2}}\left(\partial_{l} J^{12}-\partial_{r} J^{12} l^{\prime}\right) F_{21} \delta l, \\
\mathcal{L}_{F F}^{(2)} \propto \frac{1}{4} \sqrt{-E} S^{\alpha \beta} S^{r \lambda} F_{\beta \gamma} F_{\alpha \lambda,}
\end{gathered}
$$

and along $\phi$ and $\psi$ :

$$
\begin{gathered}
\mathcal{L}_{\psi \psi, \phi \phi}^{(2)} \alpha \frac{1}{2} \sqrt{-E} S^{\alpha \beta}\left(G_{\psi \psi} \partial_{\alpha} \delta \psi \partial_{\beta} \delta \psi+G_{\phi \phi} \partial_{\alpha} \delta \phi \partial_{\beta} \delta \phi\right), \\
\mathcal{L}_{\psi \phi}^{(2)} \propto(\cos \alpha) P H \delta \psi \partial_{0} \delta \phi .
\end{gathered}
$$


Here $E_{\alpha \beta}$ is the pull-back of the generalized metric on the classical D5-brane embedding:

$$
E_{\alpha \beta}=\partial_{\alpha} \bar{X}^{\mu} \partial_{\beta} \bar{X}^{v}\left(G_{\mu v}+B_{\mu v}\right)
$$

while $S^{\alpha \beta}$ and $J^{\alpha \beta}$ are the symmetric and antisymmetric elements of the inverse generalized metric $E^{\alpha \beta}$ :

$$
E^{\alpha \beta}=S^{\alpha \beta}+J^{\alpha \beta}
$$

The determinant $E$ and the function $K=P$ are given by

$$
\begin{gathered}
\sqrt{-E}=(\cos \alpha) r^{2} \sqrt{1+l^{\prime 2}} \sqrt{1+\frac{R^{4} H^{2}}{\left(r^{2}+l^{2}\right)^{2}}} \equiv g(r) \cos \alpha, \\
P=\frac{4 R^{4} r^{2} l^{2}}{\left(r^{2}+l^{2}\right)^{3}}\left(r l^{\prime}-l\right) .
\end{gathered}
$$

As one can see, the fluctuations along $\psi$ and $\phi$ decouple from the fluctuations along $l$ and the fluctuations of the gauge field $A_{\alpha}$. To study the pseudo-Goldstone modes of the dual theory, one should focus on the fluctuations along $\psi$ and $\phi$. The equations of motion derived from the quadratic action (4.115) are the following:

$$
\begin{aligned}
& \partial_{r}\left(\frac{g(r) l^{2}}{1+l^{\prime 2}} \partial_{r} \delta \psi\right)+\frac{g(r) R^{4} l^{2}}{\left(r^{2}+l^{2}\right)^{2}} \widetilde{W} \delta \psi+\frac{g(r) l^{2}}{r^{2}} \Delta_{(2)} \delta \psi-P H \partial_{0} \delta \phi=0, \\
& \partial_{r}\left(\frac{g(r) l^{2}}{1+l^{\prime 2}} \partial_{r} \delta \phi\right)+\frac{g(r) R^{4} l^{2}}{\left(r^{2}+l^{2}\right)^{2}} \widetilde{W} \delta \phi+\frac{g(r) l^{2}}{r^{2}} \Delta_{(2)} \delta \phi+P H \partial_{0} \delta \psi=0,
\end{aligned}
$$

where

$$
\widetilde{\mathrm{W}}=-\partial_{0}^{2}+\frac{\partial_{1}^{2}+\partial_{2}^{2}}{1+R^{4} H^{2} /\left(r^{2}+l^{2}\right)^{2}} .
$$

Note that the background magnetic field breaks the $S O(1,2)$ Lorentz symmetry to $S O(2)$, which manifests itself in the modified laplacian (4.120). Next one considers a plane-wave ansatz:

$$
\delta \phi=e^{i(\omega t-\vec{k} \vec{x})} \eta_{1}(r), \quad \delta \psi=e^{i(\omega t-\vec{k} \vec{x})} \eta_{2}(r)
$$


Using the ansätz (4.121), one gets

$$
\begin{aligned}
& \partial_{r}\left(\frac{g(r) l^{2}}{1+l^{\prime 2}} \eta_{1}^{\prime}\right)+\frac{g(r) R^{4} l^{2}}{\left(r^{2}+l^{2}\right)^{2}}\left(\omega^{2}-\frac{\vec{k}^{2}}{1+R^{4} H^{2} /\left(r^{2}+l^{2}\right)^{2}}\right) \eta_{1}-i \omega P H \eta_{2}=0, \\
& \partial_{r}\left(\frac{g(r) l^{2}}{1+l^{\prime 2}} \eta_{2}^{\prime}\right)+\frac{g(r) R^{4} l^{2}}{\left(r^{2}+l^{2}\right)^{2}}\left(\omega^{2}-\frac{\vec{k}^{2}}{1+R^{4} H^{2} /\left(r^{2}+l^{2}\right)^{2}}\right) \eta_{2}+i \omega P H \eta_{1}=0 .
\end{aligned}
$$

The equations of motion in (4.122) can be decoupled by the definition $\eta_{ \pm}=\eta_{1} \pm i \eta_{2}$. The result is

$$
\begin{aligned}
& \partial_{r}\left(\frac{g(r) l^{2}}{1+l^{\prime 2}} \eta_{+}^{\prime}\right)+\frac{g(r) R^{4} l^{2}}{\left(r^{2}+l^{2}\right)^{2}}\left(\omega^{2}-\frac{\vec{k}^{2}}{1+R^{4} H^{2} /\left(r^{2}+l^{2}\right)^{2}}\right) \eta_{+}-\omega P H \eta_{+}=0, \\
& \partial_{r}\left(\frac{g(r) l^{2}}{1+l^{\prime 2}} \eta_{-}^{\prime}\right)+\frac{g(r) R^{4} l^{2}}{\left(r^{2}+l^{2}\right)^{2}}\left(\omega^{2}-\frac{\vec{k}^{2}}{1+R^{4} H^{2} /\left(r^{2}+l^{2}\right)^{2}}\right) \eta_{-}+\omega P H \eta_{-}=0 .
\end{aligned}
$$

Because of the broken Lorentz symmetry, the $1+2$ dimensional mass $M^{2}=\omega^{2}-\vec{k}^{2}$ depends on the choice of frame. One can define the spectrum of excitations as the rest energy (consider the frame with $\vec{k}=0$ ). The spectrum is discrete. Furthermore, just as in the D3/D7 case there is a Zeeman splitting of the spectrum due to the external magnetic field. Interestingly, at low energy the splitting is breaking the degeneracy of the lowest energy state and as a result there is only one pseudo-Goldstone boson. This is not in contradiction with the Goldstone theorem because there is no Lorentz symmetry. This opens the possibility of having two types of Goldstone modes: type I and type II satisfying odd and even dispersion relations correspondingly. In this case, there is a modified counting rule ([41], see also [44]) which states that the number of GBs of type I plus twice the number of GBs of type II is greater than or equal to the number of broken generators. As we are going to show below the single Goldstone mode that we see satisfies a quadratic dispersion relation (hence is type II) and the modified counting rule is not violated. Note also that for large bare masses $m$ (and correspondingly large values of $l$ ), the term proportional to the magnetic field is suppressed and the meson spectrum should approximate to the result for the pure $\mathrm{AdS}_{5} \times S^{5}$ space-time case studied in $[45,46]$, where the authors obtained the following relation:

$$
\omega_{n}=\frac{2 m}{R^{2}} \sqrt{\left(n+\frac{1}{2}\right)\left(n+\frac{3}{2}\right)},
$$

between the eigenvalue of the $n$th excited state $\omega_{n}$ and the bare mass $m$.

In order to obtain the meson spectrum, one solves numerically the equations of motion (4.123) in the rest frame $(\vec{k}=0)$. The quantization condition for the spectrum comes from imposing regularity at infinity. More precisely one requires that $\eta_{ \pm} \sim 1 / r$ at infinity $(r \rightarrow \infty)$. The results are summarized in Figure 9. It is convenient to define the dimensionless quantities $\tilde{m}=m / R \sqrt{H}$ and $\tilde{\omega}=\omega R / \sqrt{H}$. As one can see from Figure 9, for large $\tilde{m}$ the spectrum asymptotes to that of pure $\mathrm{AdS}_{5} \times S^{5}$, given by (4.124). The Zeeman splitting of the spectrum is also evident. It is interesting that as a result of the splitting of the ground state there is only 


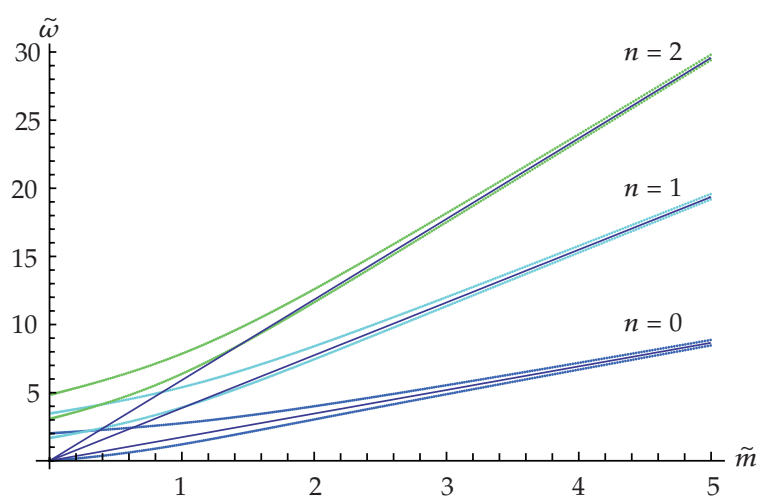

Figure 9: The meson spectrum of the first three excited states is plotted. There is Zeeman splitting of the spectrum and the existence of a mass gap at $\tilde{m}=0$ as well as a single Goldstone boson mode. For large $\tilde{m}$ the spectrum asymptotes to that of zero magnetic field given by (4.63) (straight lines).

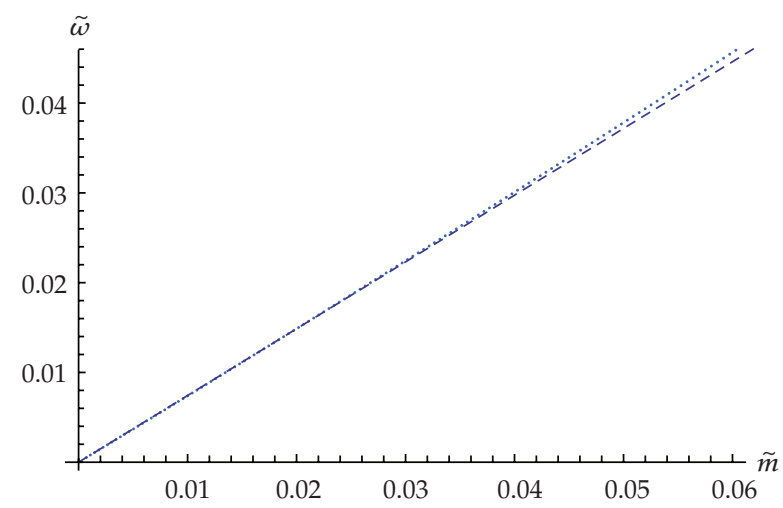

Figure 10: Plot of the spectrum of the ground state from Figure 9 for small bare masses. The dashed line corresponds to the linear behavior from (4.125).

a single pseudo-Goldstone mode. Furthermore, as can be seen from Figure 10, for small bare masses instead of the usual Gell-Mann-Oakes-Renner relation, there is a linear dependence $\tilde{\omega} \sim \tilde{m}$. As we will review in the next subsection, the slope is given by the relation:

$$
\tilde{\omega}=\frac{4 \widetilde{c}_{\mathrm{cr}}}{\pi} \tilde{m} \approx 0.736 \tilde{m}
$$

It is also interesting to study the dispersion relation of the Goldstone mode. Since the Lorentz symmetry is broken and only one pseudo-Goldstone mode is observed, one anticipates a quadratic dispersion relation (see [44, 47] for discussion).

In order to obtain the dispersion relation of the Goldstone mode, the authors of [12] numerically solve (4.123) at very small bare mass $\tilde{m} \approx 0.0007$ and for a range of small 


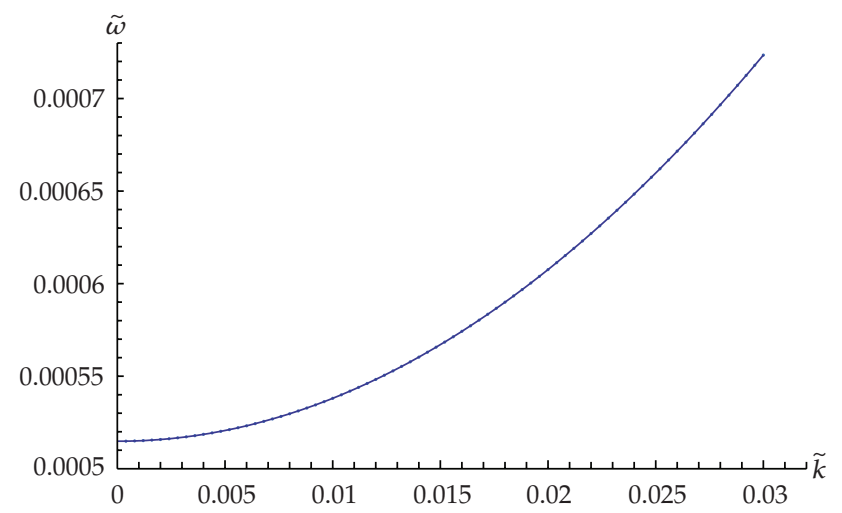

Figure 11: Plot of the dispersion relation of the pseudo-Goldstone mode for $\tilde{m} \approx 0.0007$. The parabolic fit corresponds to (4.128).

momenta $\tilde{\vec{k}}=\vec{k} R / \sqrt{H}$. The result is presented in Figure 11. There is indeed a quadratic dispersion relation. It has been shown [12] that the dispersion relation is given by

$$
\tilde{\omega}=\gamma \widetilde{\vec{k}}^{2}+\frac{4}{\pi} \widetilde{c}_{\mathrm{cr}} \tilde{m}
$$

where

$$
r=\frac{4}{\pi} \int_{0}^{\infty} d \widetilde{r} \frac{\widetilde{r}^{2} \widetilde{l}^{2} \sqrt{1+\widetilde{l}^{2}}}{\left(\tilde{r}^{2}+\tilde{l}^{2}\right) \sqrt{1+\left(\tilde{r}^{2}+\widetilde{l}^{2}\right)^{2}}}
$$

For $\tilde{m} \approx 0.0007$, the relation (4.126) is given by

$$
\tilde{\omega} \approx 0.232 \widetilde{\vec{k}}^{2}+0.000515
$$

and is represented by the fitted curve in Figure 11.

\section{Low Energy Dispersion Relation}

In order to obtain the dispersion relation for the pseudo-Goldstone mode, one considers [12] the limit of small $\omega$ in the first equation in (4.123) thus leaving only the linear potential term in $\widetilde{\omega}$. In view of the observed quadratic dispersion relation (4.126), one also keeps the $\vec{k}^{2}$ term in (4.123).

$$
\partial_{r}\left(\frac{g(r) l^{2}}{1+l^{\prime 2}} \eta_{+}^{\prime}\right)-\left(\omega P H+\frac{g(r) R^{4} l^{2}}{\left(r^{2}+l^{2}\right)^{2}+R^{4} H^{2}} \vec{k}^{2}\right) \eta_{+}=0
$$


It is convenient to define the following variables:

$$
\Theta^{2}=\frac{g(r) l^{2}}{1+l^{\prime 2}}, \quad \xi=\eta_{+} \Theta
$$

Then (4.129) can be written as

$$
\ddot{\xi}-\frac{\ddot{\Theta}}{\Theta} \xi-\left(\omega P H+\frac{g(r) R^{4} l^{2}}{\left(r^{2}+l^{2}\right)^{2}+R^{4} H^{2}} \vec{k}^{2}\right) \frac{\xi}{\Theta^{2}}=0,
$$

where the overdots represent derivatives with respect to $r$. Now if one takes the limit $m \rightarrow 0$ one has that $\omega \rightarrow 0$ and $k \rightarrow 0$ and obtains that

$$
\xi=\left.\Theta\right|_{\omega=0} \equiv \bar{\Theta}
$$

is a solution to (4.131). The next step is to consider small $m$ and expand

$$
\xi=\bar{\Theta}+\delta \xi, \quad \Theta=\bar{\Theta}+\delta \Theta
$$

where the variations $\delta \xi$ and $\delta \Theta$ are vanishing in the $m \rightarrow 0$, limit. Then, to, leading order in $m$ (keeping in mind that $\omega \sim m$ and $\vec{k}^{2} \sim m$ ) one obtains

$$
\delta \ddot{\xi}-\frac{\ddot{\bar{\Theta}}}{\bar{\Theta}} \delta \xi-\delta\left(\frac{\ddot{\Theta}}{\bar{\Theta}}\right) \bar{\Theta}-\left(\omega P H+\frac{g(r) R^{4} l^{2}}{\left(r^{2}+l^{2}\right)^{2}+R^{4} H^{2}} \vec{k}^{2}\right) \frac{1}{\bar{\Theta}}=0
$$

Now one multiplies (4.134) by $\bar{\Theta}$ and integrates along $r$. The result is

$$
\left.(\bar{\Theta} \delta \dot{\xi}-\dot{\bar{\Theta}} \delta \xi)\right|_{0} ^{\infty}-\left.(\bar{\Theta} \delta \dot{\Theta}-\dot{\bar{\Theta}} \delta \Theta)\right|_{0} ^{\infty}-\omega H \int_{0}^{\infty} d r P(r)-\frac{\pi}{4} R^{5} \sqrt{H} \gamma \vec{k}^{2}=0
$$

Using the definitions of $\Theta, P(r)$ and $\xi$ and requiring regularity at infinity for $\eta_{+}$, one can show that the first term in (4.135) vanishes and that

$$
\left.(\bar{\Theta} \delta \dot{\Theta}-\dot{\bar{\Theta}} \delta \Theta)\right|_{0} ^{\infty}=c \delta m, \quad \int_{0}^{\infty} d r P(r)=-\frac{R^{4} \pi}{4},
$$

And hence using the previous definitions, $\tilde{m}=m / R \sqrt{H}, \tilde{c}=c / R^{2} H, \tilde{\omega}=\omega R / \sqrt{H}$, and $\widetilde{\vec{k}}=\vec{k} R / \sqrt{H}$, one obtains (4.126).

\section{Effective Chiral Lagrangian}

In order to obtain the $1+2$ dimensional effective action describing the pseudo-Goldstone mode, one considers [12] the $1+5$ dimensional action (4.115) for a classical embedding in 
the vicinity of the critical embedding, namely, that embedding corresponding to a very small bare mass $\tilde{m}$. Next one takes the ansätz for the fields $\delta \phi$ and $\delta \psi$ :

$$
\delta \phi=\frac{\xi_{1}(r)}{\Theta(r)} \chi_{1}(x), \quad \delta \psi=\frac{\xi_{2}(r)}{\Theta(r)} \chi_{2}(x) .
$$

Since one is close to the critical embedding, one considers the same expansion as in equation (4.133):

$$
\xi_{i}=\bar{\Theta}+\delta \xi_{i}, \quad i=1 \quad \text { or } 2, \quad \Theta=\bar{\Theta}+\delta \Theta
$$

By definition, it follows that as $\tilde{m} \rightarrow 0, \delta \xi_{i}$ and $\delta \Theta$ vanish. Then to leading order, one has that

$$
\begin{aligned}
& \partial_{r} \delta \phi=\frac{1}{\bar{\Theta}^{2}}\left[\left(\bar{\Theta} \delta \dot{\xi}_{1}-\dot{\bar{\Theta}} \delta \xi_{1}\right)+(\dot{\bar{\Theta}} \delta \Theta-\bar{\Theta} \delta \dot{\Theta})\right] x_{1}(x), \quad \partial_{\mu} \delta \phi=\partial_{\mu} \chi_{1}(x), \quad \mu=0,1,2, \\
& \partial_{r} \delta \psi=\frac{1}{\bar{\Theta}^{2}}\left[\left(\bar{\Theta} \delta \dot{\xi}_{2}-\dot{\bar{\Theta}} \delta \xi_{2}\right)+(\dot{\bar{\Theta}} \delta \Theta-\bar{\Theta} \delta \dot{\Theta})\right] x_{2} t(x), \quad \partial_{\mu} \delta \psi=\partial_{\mu} \chi_{2}(x), \quad \mu=0,1,2 .
\end{aligned}
$$

Next, one integrates (4.115) along $r$ from $0, \infty$ and along the internal unit sphere $\widetilde{\Omega}_{2}$. The interesting term is the part of the kinetic term involving derivatives along $r$. After integration by parts, it boils down to a mass term for the $1+2$ dimensional fields $\chi_{1}, \chi_{2}$. Explicitly

$$
\begin{aligned}
\int d r d \widetilde{\Omega}_{2} \frac{1}{2} \frac{g(r) l^{2}}{1+l^{\prime 2}} \partial_{r} \delta \phi \partial_{r} \delta \phi & =-\int d r d \widetilde{\Omega}_{2} \frac{1}{2} \partial_{r}\left(\frac{g(r) l^{2}}{1+l^{\prime 2}} \partial_{r} \delta \phi\right) \delta \phi \\
& =-\left.4 \pi\left[\left(\bar{\Theta} \delta \dot{\xi}_{1}-\dot{\bar{\Theta}} \delta \xi_{1}\right)+(\dot{\bar{\Theta}} \delta \Theta-\bar{\Theta} \delta \dot{\Theta})\right]\right|_{0} ^{\infty} \frac{1}{2} x_{1}^{2} \\
& =4 \pi m c \frac{1}{2} x_{1}^{2}
\end{aligned}
$$

Here the same arguments as in (4.135) have been used. It is clear that one can perform an analogous calculation for the term involving $\partial_{r} \delta \psi$. The rest of the terms are dealt with straightforwardly by integrating along $r$. The resulting action is

$$
\frac{S_{\mathrm{eff}}}{\left(2 \pi \alpha^{\prime}\right)^{2}}=\int d^{3} x\left\{\frac{f_{\pi \|}^{2}}{4} \partial_{0} \chi^{*} \partial_{0} \chi-\frac{f_{\pi \perp}^{2}}{4} \partial_{i} \chi^{*} \partial_{i} \chi-\mu \frac{i}{2}\left(\chi \partial_{0} \chi^{*}-\chi^{*} \partial_{0} \chi\right)+\frac{m_{q}}{2}\langle\bar{\Psi} \Psi\rangle_{0} \chi^{*} X\right\},
$$


where the complex scalar field $x=x_{1}+i x_{2}$ has been defined. The constants in the effective action are given by

$$
\begin{gathered}
\frac{f_{\pi \|}^{2}}{4}=\frac{N}{2} \int_{0}^{\infty} d r \frac{g(r) R^{4} l^{2}}{\left(r^{2}+l^{2}\right)^{2}}, \quad \frac{f_{\pi \perp}^{2}}{4}=\frac{N}{2} \int_{0}^{\infty} d r \frac{g(r) R^{4} l^{2}}{\left(r^{2}+l^{2}\right)^{2}+R^{4} H^{2}}, \\
\mu=\frac{N}{8} \pi R^{4} H, \quad\langle\bar{\psi} \psi\rangle=-\left(2 \pi \alpha^{\prime}\right) N c_{\mathrm{cr}}, \quad N=4 \pi N_{f} \frac{\mu_{5}}{g_{s}}, \quad m_{q}=\frac{m}{2 \pi \alpha^{\prime}} .
\end{gathered}
$$

\subsection{Summary}

In this section, we reviewed the studies of the influence of external magnetic field on holographic gauge theories dual to the D3/D7- and the D3/D5-intersections.

In the case of the D3/D7-system, we described the effect of mass generation realized as a separation of the probe D7-branes and the background D3-branes in the interior of the geometry. The review of the study of the meson spectrum revealed Zeeman splitting and the existence of Goldstone mode associated to the spontaneously broken Chiral Symmetry. In the limit of small bare masses, we reviewed the analytic derivation of the effective chiral action obtained in [12] after dimensional reduction of the eight-dimensional effective action of the probe D7-brane. As expected the mass of the pseudo-Goldstone modes satisfies the Gell-Mann-Oaks-Renner relation. The effective action also suggests an anisotropic relativistic dispersion relation consistent with the residual $S O(1,1) \times S O(2)$ symmetry. An integral expression for the parameter of anisotropy is also obtained. It would be particularly interesting if one could obtain this parameter via alternative non-perturbative approach, such as lattice simulation.

The D3/D5-system studied in Section 4.2 exhibit properties similar to the D3/D7 system. Again there is a mass generation realized as separation of the color and flavor branes. The meson spectrum also has Zeeman splitting and a Goldstone mode. There is however a crucial difference. Due to the completely broken Lorentz symmetry, the goldstone modes satisfy nonrelativistic dispersion relation and modified counting rule. An integral expression for the Galilean mass in terms of the parameter $\gamma$ given in (4.127) has also been obtained. It would be nice if one can compare this result with a result obtained via lattice simulation.

Overall the holographic studies that we presented in this section confirm the universal nature of the phenomenon of magnetic catalysis of chiral symmetry breaking reviewed in Section 2.2. It is also somewhat satisfying that some of the results (such as the Gell-MannOaks-Renner relation) presented here have been obtained in a closed form and are consistent with the results derived via standard chiral dynamics.

\section{Conclusion}

In this paper, we outlined a recent application of the AdS/CFT correspondence to study the effect of magnetic catalysis of mass generation in holographic gauge theories dual to the D3/D5- and the D3/D7-intersections. Our goal was to illustrate the potential of the correspondence to capture essential properties of the strongly coupled regime of non-abelian gauge theories especially when the investigated phenomenon is of a universal nature. We attempted to give the review a self-contained and somewhat pedagogical form. 
The purpose of Section 2 was to remind the reader about some basic properties of flavored Yang-Mills theories as well as to provide the physical motivation for the holographic studies subject to our investigation. We provided a brief description of the global symmetries of the theory with emphasis on the anomalous and nonsinglet Chiral Symmetries. We also outlined the effective chiral lagrangian description of the effect of Chiral Symmetry breaking and provided a short derivation of the Gell-Mann-Oaks-Renner relation. The second part of Section 2 describes the standard field theory approach to studying the phenomenon of magnetic catalysis of mass generation.

In Section 3 of the paper, we outlined the basics of the AdS/CFT correspondence. We started with a review of the general ideas that lead to its formulation and descried in details the physical justification of the correspondence in the framework of string theory. We also provided a brief description of the way the standard AdS/CFT dictionary operates. In the second part of Section 3, we focused on the addition of flavor degrees to the correspondence. We reviewed the standard way [3] to introduce quenched fundamental matter by adding probe flavor branes to the dual supergravity background. We provided some basic extracts from the generalized AdS/CFT dictionary which are implemented in the holographic set up reviewed in Section 4.

Section 4 is the main part of the review. We reviewed the holographic study of the influence of external magnetic field on holographic gauge theories dual to the D3/D5- and the D3/D7-intersections. We reviewed the general properties of the holographic set up. We described how spontaneous symmetry breaking is realized as a separation between the flavor and color branes in the bulk of the geometry. Investigations of the meson spectrum revealed Zeeman splitting of the energy level as well as the existence of Goldstone modes. In the case of the D3/D7-set up, we reviewed the analytic derivation of the Gell-Mann-Oaks-Renner relation performed in [12]. We also investigated the dispersion relations of the pseudoGoldosten modes and verified that they are consistent with the residual space time symmetry: $S O(1,1) \times S O(2)$ in the D3/D7 set up and $S O(2)$ in the D3/D5 set up. It is intriguing that the D3/D5 system exhibits a nonrelativistic dispersion relation.

The overall goal of the review was to review in a self-consistent way one of the many successful applications of the AdS/CFT duality to the investigation of universal properties of strongly coupled Yang-Mills theories. The results presented in Section 4 seem to be perfect agreement with the proposed universal nature of the effect of chiral symmetry breaking in an external magnetic field. We hope that future investigations using alternative nonperturbative techniques such as lattice simulations could confirm qualitatively the results presented in Section 4. Such studies could provide a non-trivial check of the AdS/CFT correspondence in this essentially non-supersymmetric set up.

\section{Acknowledgments}

Veselin Filev would like to thank Clifford V. Johnson and Jonathan P. Shock for fruitful collaboration in $[10,12]$. The work of Veselin Filev was supported by an IRCSET postdoctoral fellowship. The work of Radoslav C. Raskov was supported in part by the Austrian Research Fund FWF no. P22000, NSFB VU-F-201/06, and DO 02-257.

\section{References}

[1] J. Maldacena, "The large $N$ limit of superconformal field theories and supergravity," Advances in Theoretical and Mathematical Physics, vol. 2, no. 2, pp. 231-252, 1998. 
[2] J. Maldacena, "The large- $N$ limit of superconformal field theories and supergravity," International Journal of Theoretical Physics, vol. 38, no. 4, pp. 1113-1133, 1999.

[3] A. Karch and E. Katz, "Adding flavor to AdS/CFT," Journal of High Energy Physics, vol. 2002, no. 6, article 043, 2002.

[4] S. S. Gubser and A. Karch, "From Gauge-string duality to strong interactions: a Pedestrian's guide," Annual Review of Nuclear and Particle Science, vol. 59, pp. 145-168, 2009.

[5] V. P. Gusynin, V. A. Miransky, and I. A. Shovkovy, "Dimensional reduction and dynamical chiral symmetry breaking by a magnetic field in $3+1$ dimensions," Physics Letters B, vol. 349, no. 4, pp. 477-483, 1995.

[6] K. G. Klimenko, "The three-dimensional Gross-Neveu model in an external magnetic field. I," Theoretical and Mathematical Physics, vol. 89, no. 2, pp. 1161-1169, 1991, translated in Teoreticheskaya i Matematicheskaya Fizika, vol. 89, no. 2, pp. 211-221, 1991.

[7] K. G. Klimenko, "Three-dimensional Gross-Neveu model at nonzero temperature and in the presence of an external electromagnetic field," Zeitschrift für Physik C, vol. 54, no. 2, pp. 323-329, 1992.

[8] K. G. Klimenko, "The three-dimensional Gross-Neveu model at nonzero temperature and in an external magnetic field," Theoretical and Mathematical Physics, vol. 90, no. 1, pp. 1-6, 1992, translated in Teoreticheskaya i Matematicheskaya Fizika, vol. 90, no. 1, pp. 3-11, 1992.

[9] M. Gell-Mann, R. J. Oakes, and B. Renner, "Behavior of current divergences under SU3×SU3," Physical Review, vol. 175, no. 5, pp. 2195-2199, 1968.

[10] V. G. Filev, C. V. Johnson, R. C. Rashkov, and K. S. Viswanathan, "Flavoured large N gauge theory in an external magnetic field," Journal of High Energy Physics, vol. 2007, no. 10, article 019, 2007.

[11] V. G. Filev, "Criticality, scaling and chiral symmetry breaking in external magnetic field," Journal of High Energy Physics, vol. 2008, no. 4, article 088, 2008.

[12] V. G. Filev, C. V. Johnson, and J. P. Shock, "Universal holographic chiral dynamics in an external magnetic field," Journal of High Energy Physics, vol. 2009, no. 8, article 013, 2009.

[13] J. Erdmenger, R. Meyer, and J. P. Shock, "AdS/CFT with flavour in electric and magnetic KalbRamond fields," Journal of High Energy Physics, vol. 2007, no. 12, article 091, 2007.

[14] A. V. Smilga, Lectures on Quantum Chromodynamics, World Scientific, Singapore, 2001.

[15] E. Witten, "Current algebra theorems for the U(1) "Goldstone boson"," Nuclear Physics B, vol. 156, no. 2, pp. 269-283, 1979.

[16] G. Veneziano, “U(1) without instantons," Nuclear Physics B, vol. 159, no. 1-2, pp. 213-224, 1979.

[17] J. L. F. Barbón, C. Hoyos, D. Mateos, and R. C. Myers, "The holographic life of the $\eta^{\prime}$," Journal of High Energy Physics, vol. 2004, no. 10, article 029, 2004.

[18] V. P. Gusynin, V. A. Miransky, and I. A. Shovkovy, “Catalysis of dynamical flavor symmetry breaking by a magnetic field in $2+1$ dimensions," Physical Review Letters, vol. 73, no. 26, pp. 3499-3502, 1994.

[19] V. P. Gusynin, V. A. Miransky, and I. A. Shovkovy, "Erratum: catalysis of dynamical flavor symmetry breaking by a magnetic field in $2+1$ dimensions," Physical Review Letters, vol. 76, no. 6, p. 1005, 1996.

[20] G. 't. Hooft, "A planar diagram theory for strong interactions," Nuclear Physics B, vol. 72, no. 3, pp. 461-473, 1974.

[21] C. V. Johnson, D-Branes, Cambridge Monographs on Mathematical Physics, Cambridge University Press, Cambridge, UK, 2003.

[22] O. Aharony, S. S. Gubser, J. Maldacena, H. Ooguri, and Y. Oz, "Large N field theories, string theory and gravity," Physics Reports, vol. 323, no. 3-4, pp. 183-386, 2000.

[23] R. G. Leigh, "Dirac-born-infeld action from Dirichlet sigma model," Modern Physics Letters A, vol. 4, no. 28 , pp. 2767-2772, 1989.

[24] S. S. Gubser, I. R. Klebanov, and A. A. Tseytlin, "String theory and classical absorption by threebranes," Nuclear Physics B, vol. 499, no. 1-2, pp. 217-240, 1997.

[25] I. R. Klebanov, “World-volume approach to absorption by non-dilatonic branes," Nuclear Physics B, vol. 496, no. 1-2, pp. 231-242, 1997.

[26] J. Erdmenger, N. Evans, I. Kirsch, and E. J. Threlfall, "Mesons in gauge/gravity duals," European Physical Journal A, vol. 35, no. 1, pp. 81-133, 2008.

[27] S. S. Gubser, I. R. Klebanov, and A. M. Polyakov, "Gauge theory correlators from non-critical string theory," Physics Letters B, vol. 428, no. 1-2, pp. 105-114, 1998.

[28] E. Witten, "Anti de Sitter space and holography," Advances in Theoretical and Mathematical Physics, vol. 2, no. 2, pp. 253-291, 1998 
[29] D. Z. Freedman, S. D. Mathur, A. Matusis, and L. Rastelli, "Correlation functions in the $\mathrm{CFT}_{d} / \mathrm{AdS}_{d+1}$ correspondence," Nuclear Physics B, vol. 546, no. 1-2, pp. 96-118, 1999.

[30] S. Lee, S. Minwalla, M. Rangamani, and N. Seiberg, "Three-point functions of chiral operators in $D=4, N=4$ SYM at large $N, "$ Advances in Theoretical and Mathematical Physics, vol. 2, no. 4, pp. 697-718, 1998.

[31] D. Berenstein, J. Maldacena, and H. Nastase, "Strings in flat space and pp waves from $\mathcal{N}=4$ super Yang Mills," Journal of High Energy Physics, vol. 2002, no. 4, article 013, 2002.

[32] N. Beisert, "The dilatation operator of $N=4$ super Yang-Mills theory and integrability," Physics Reports, vol. 405, no. 1-3, pp. 1-202, 2004.

[33] J. C. Plefka, "Lectures on the plane-wave string/gauge theory duality," Fortschritte der Physik, vol. 52, no. 2-3, pp. 264-301, 2004.

[34] M. Kruczenski, D. Mateos, R. C. Myers, and D. J. Winters, "Meson spectroscopy in AdS/CFT with flavour," Journal of High Energy Physics, vol. 2003, no. 7, article 049, 2003.

[35] A. Karch, A. O’Bannon, and K. Skenderis, "Holographic renormalization of probe D-branes in AdS/CFT," Journal of High Energy Physics, vol. 2006, no. 4, article 015, 2006.

[36] J. Babington, J. Erdmenger, N. Evans, Z. Guralnik, and I. Kirsch, "Chiral symmetry breaking and pions in nonsupersymmetric gauge/gravity duals," Physical Review D, vol. 69, no. 6, Article ID 066007, 2004

[37] M. Kruczenski, D. Mateos, R. C. Myers, and D. J. Winters, “Towards a holographic dual of large- $\mathrm{N}_{c}$ QCD," Journal of High Energy Physics, vol. 2004, no. 5, article 041, 2004.

[38] N. Evans and J. P. Shock, "Chiral dynamics from AdS space," Physical Review D, vol. 70, Article ID 046002, 11 pages, 2004.

[39] R. C. Myers, A. O. Starinets, and R. M. Thomson, "Holographic spectral functions and diffusion constants for fundamental matter," Journal of High Energy Physics, vol. 2007, no. 11, article 091, 2007.

[40] D. Areán, A. Paredes, and A. V. Ramallo, "Adding flavor to the gravity dual of non-commutative gauge theories," Journal of High Energy Physics, vol. 2005, no. 8, article 017, 2005.

[41] H. B. Nielsen and S. Chadha, "On how to count Goldstone bosons," Nuclear Physics B, vol. 105, no. 3, pp. 445-453, 1976.

[42] D. Mateos, R. C. Myers, and R. M. Thomson, "Thermodynamics of the brane," Journal of High Energy Physics, vol. 2007, no. 5, artilce 067, 2007.

[43] D. Mateos, R. C. Myers, and R. M. Thomson, "Holographic phase transitions with fundamental matter," Physical Review Letters, vol. 97, no. 9, Article ID 091601, 4 pages, 2006.

[44] T. Brauner, "Goldstone boson counting in linear sigma models with chemical potential," Physical Review D, vol. 72, Article ID 076002, 10 pages, 2005.

[45] D. Areán and A. V. Ramallo, "Open string modes at brane intersections," Journal of High Energy Physics, vol. 2006, no. 4, article 037, 2006.

[46] R. C. Myers and R. M. Thomson, "Holographic mesons in various dimensions," Journal of High Energy Physics, vol. 2006, no. 9, article 066, 2006.

[47] T. Brauner, "Goldstone bosons in presence of charge density," Physical Review D, vol. 75, Article ID 105014, 13 pages, 2007. 

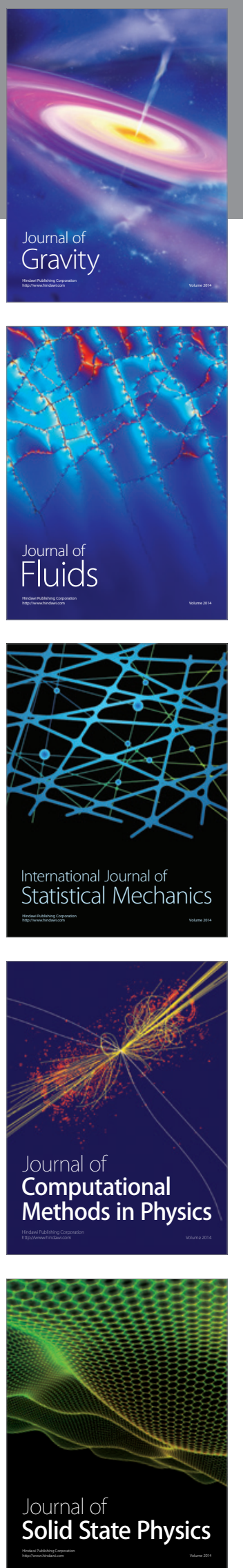

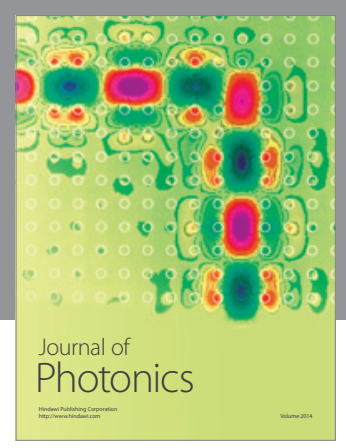

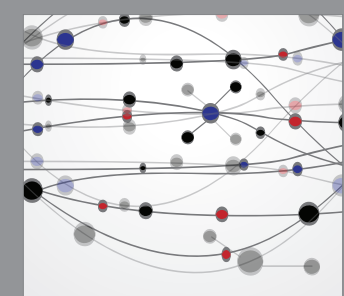

The Scientific World Journal
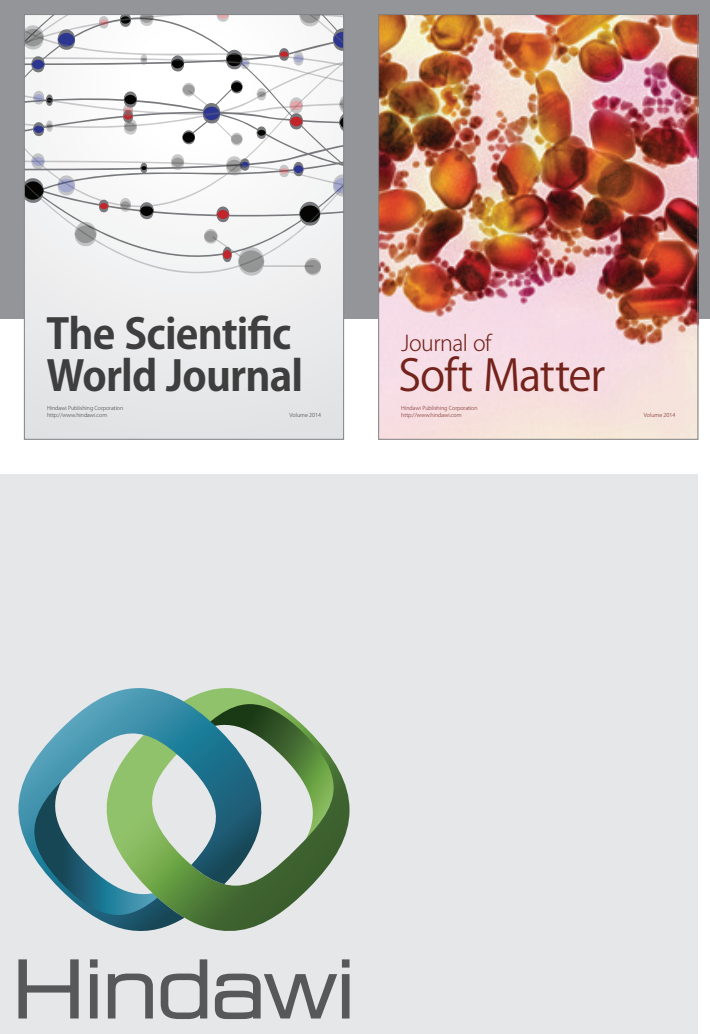

Submit your manuscripts at

http://www.hindawi.com
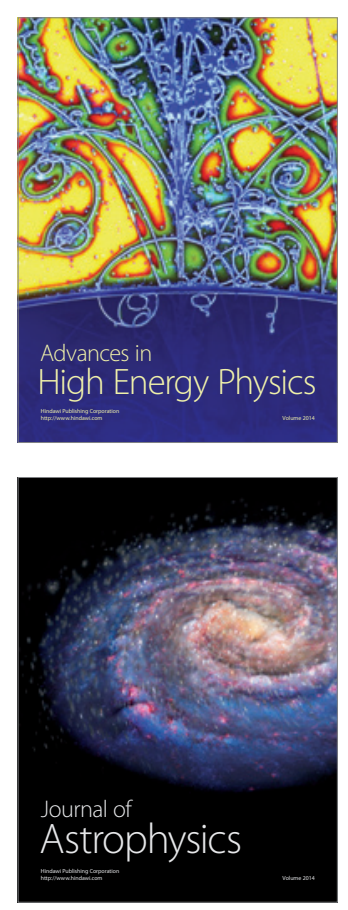
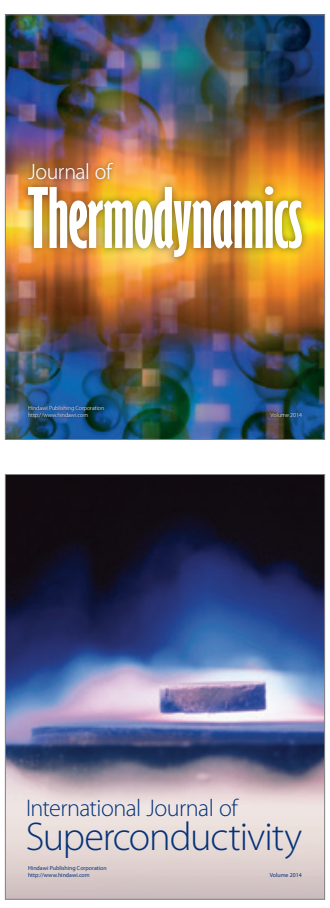
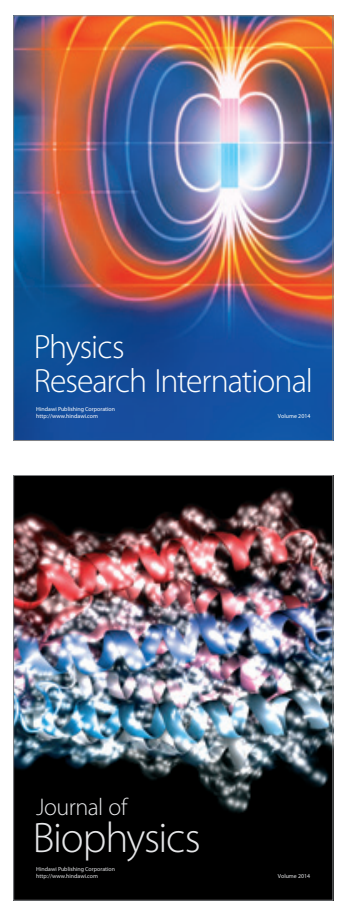
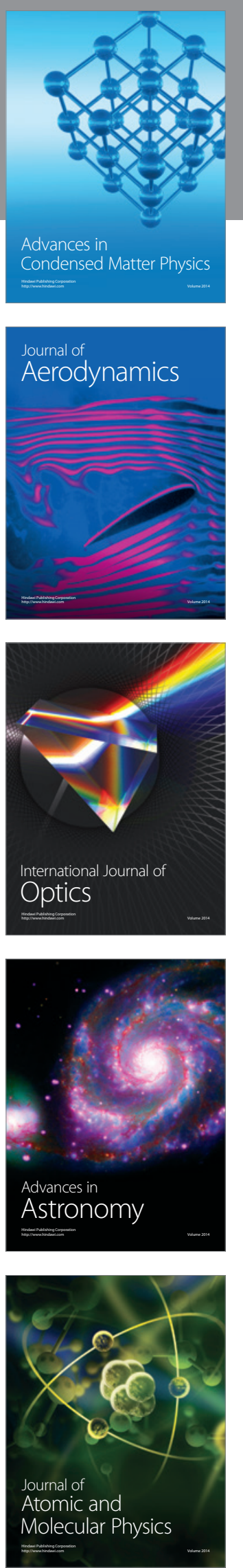